\title{
Optical determination of temperature and concentrations of homogeneous turbulent gas mixtures
}

\author{
Tao Ren, Michael F. Modest*
}

School of Engineering, University of California, Merced, California, USA

\begin{abstract}
In this study, we present an inverse radiation model to reconstruct time-averaged temperature, species concentrations and their root mean square (rms) values for turbulent gas mixtures, based on line-of-sight spectral transmissivity measurements. Time-averaged transmissivities and their rms values are successfully related to timeaveraged temperatures, species concentrations and their rms values by considering interactions between turbulence and radiation (TRI). The turbulence length scale is retrieved simultaneously with the turbulent scalars. Optimal wavenumber ranges for retrieving temperature and species concentrations of the gas mixtures are discussed. To validate the models, measured spectra are synthesized by calculations from HITEMP 2010 for turbulent gas mixtures of $\mathrm{CO}_{2}, \mathrm{H}_{2} \mathrm{O}$ and $\mathrm{CO}$.
\end{abstract}

Keywords: mixture, temperature, concentration, turbulent, transmissivity

\section{Nomenclature}

C correlation function, -

$L \quad$ length of gas column, $\mathrm{cm}$

$P \quad$ total pressure, bar

$s \quad$ distance along path, $\mathrm{cm}$

$t \quad$ total time interval, $\mathrm{s}$

$t_{e} \quad$ turbulence integral time scale, $\mathrm{s}$

$T$ temperature, $\mathrm{K}$

$W \quad$ sensitivity coefficient, -

$x \quad$ concentration by volume, -

$\mathbf{z}$ vector of parameter scalars

\section{Greek Symbols}

$\eta \quad$ wavenumber, $\mathrm{cm}^{-1}$

$\kappa \quad$ absorption coefficient, $\mathrm{cm}^{-1}$

$\tau$ transmissivity, -

$\Gamma \quad$ pixel response function (PRF), -

$\Lambda \quad$ turbulence integral length scale, $\mathrm{cm}$

\section{Introduction}

Advanced optical diagnostics and multi-scale simulation tools will play a central role in the development of next-generation clean and efficient combustion systems, as well as in upcoming high-temperature alternative

\footnotetext{
*Corresponding author

Email address: mmodest@ucmerced.edu (Michael F. Modest)
} 
energy applications. Combustion diagnostics have reached high levels of refinement, but it remains difficult to make quantitatively accurate nonintrusive measurements of temperature and species concentrations in realistic combustion environments. Griffith et al. [1, 2] were the first to recognize that measurements of the transmissivity or emissivity of rotational spectral lines of a gas can reveal its temperature. In order to extract temperature, a nonlinear least-square method was used to fit the integrated transmission minima. In their experiments, transmissivities for $\mathrm{CO}_{2} 10.4 \mu \mathrm{m}$ and $9.4 \mu \mathrm{m}$ bands at a fine resolution of $0.29 \mathrm{~cm}^{-1}$ for pure $\mathrm{CO}_{2}$ [2] were measured. Best et al. [3, 4] combined tomography and Fourier transform infrared (FTIR) spectrometer transmission and emission spectra to extract temperature, concentration and soot volume fraction fields. Not much detail was given, except that low resolution $\left(32 \mathrm{~cm}^{-1}\right)$ scans were used. Song et al. [5-8] developed a spectral remote sensing technique to reconstruct temperature profiles in $\mathrm{CO}_{2}$ mixtures based on radiative intensity measurements. In their experiments, spectra from $1.3 \mu \mathrm{m}$ to $4.8 \mu \mathrm{m}$ were imaged onto a 160-element lead selenide array detector. Spectral information for only the $\mathrm{CO}_{2} 4.3 \mu \mathrm{m}$ band was used to retrieve the temperature profile and the spectral resolution is coarse and not changeable. For a turbulent system, it has long been recognized that the nonlinear interaction between turbulence and radiation (TRI) has profound effects on turbulent combustion [9-13]. The radiative signal from combustion gases is influenced by nonlinear interaction with turbulence. In the presence of TRI, temperature and concentration must be deduced using knowledge of turbulence structures or employing TRI models.

Work has been done to extract temperature and species concentration for laminar gaseous media containing a single emitting species [3-8, 14-17] based on optical measurements of transmission or emission. Lim et al. [18] linearized the radiative transfer equation (RTE) in conjunction with the maximum likelihood estimation to deconvolute the spectral emitted intensities for temperature and concentrations of two major combustion species $\left(\mathrm{CO}_{2}\right.$ and $\left.\mathrm{H}_{2} \mathrm{O}\right)$ and soot. The spectral emitted intensity was approximated as a linear function of temperature, soot and gas concentrations. Coefficients for the linearized function were obtained from the RADCAL database [19], a narrow-band database. The method was first verified using synthetic spectral data points in the 1.3 to $4.8 \mu \mathrm{m}$ spectral interval. At the same time, the spectral radiation intensities emitted from a onedimensional McKenna burner [20] was measured at multiple wavelengths using the high-speed mid-infrared spectrometer ES100 [21]. The estimated gas temperature and concentrations obtained using this method are very close to those obtained with thin filament pyrometry and theoretical calculations.

Experimental investigations by Faeth and Gore [22 35] and probability density function (pdf) based calculations [36-40] have shown that TRI always increases the heat loss from a flame, and this additional heat loss can reach $60 \%$ of the total and more, leading to a reduction in the local gas temperature of $200^{\circ} \mathrm{C}$ or more. Therefore, the radiative signal hitting a detector is influenced by the nonlinear interaction with turbulence. The TRI effects, although acknowledged and qualitatively understood over the last three decades or so, are extremely difficult to model. Most work on TRI has been devoted to the study of the impact of turbulence on total radiative heat transfer emitted by a hot medium. A rather different challenge is accurate modeling of the correlation between local instantaneous radiation intensity along the optical path and local absorption coefficients [41]. Most works have neglected this correlation based on the arguments given by Kabashnikov and Myasnikova [42] that, if the mean free path for radiation is much larger than the turbulence length scale, then the local intensity is governed by fluctuations far away, and thus should be only weakly correlated with local absorption coefficient fluctuations. This assumption appears to be valid over most of the gas spectrum for small-scale, lower-sooting flames and is known as the optically thin fluctuation assumption (OTFA), but questionable for very strong spectral lines. Ko et al. [43] developed a spectral remote sensing method to retrieve mean temperature and concentration from spectral turbulent intensities using the $\mathrm{CO}_{2} 4.3 \mu \mathrm{m}$ band by applying the OTFA. For their proposed method, it is claimed that the coupled temperature/concentration fluctuation amplitudes and mean values can be successfully inverted from optically measured intensity spectra. However, only retrieved mean temperature/concentration profiles along the path were presented and not much detail was given for the inverse method. Unlike modeling TRI for spectral intensity, TRI effects on transmissivity can be accurately modeled by assuming the pdf shape of the absorption coefficient. An early study by Foster [44] showed that calculation of the mean transmissivity from a turbulent flame must take turbulent fluctuations into account. Coelho [41] showed that, in the presence of turbulent fluctuations, the turbulent fluctuation of the absorption coefficient increases the transmissivity of the medium if the pdf of the absorption coefficient is Gaussian. His observation is in agreement with the theoretical findings of Foster [44]. 
Optical diagnostics based on radiometric measurements are concerned with reconstructing the spatial distribution of temperature, species concentrations and other parameters inside absorbing combustion gases. These retrieval techniques based on radiative measurements are known as inverse radiative heat transfer analyses [45]. First, a forward calculation is required to predict the detector reading as a function of the retrieval parameters of the system. Second, an objective function is formulated which represents the difference between the predicted and measured data. And third, an inverse model is used to provide updated system parameters by minimization of the objective function [46, 47]. The most robust methods for minimizing the objective function have proved to be gradient-based [48]. Steepest descent is the simplest method and can guarantee convergence, but it is known to have a very slow convergence rate [49]. Quasi-Newton methods are alternatives to Newton's method, which are used to find local minima of the objective functions avoiding to calculate the exact Hessian matrix [45, 49], which can be extremely expensive at times. In this method, at each iteration the objective function is approximated by a quadratic function and takes a step toward the minimum of the quadratic function. If the objective function is quadratic or near-quadratic, the local minimum can be found in very few steps. One of the disadvantages is that this method does not guarantee convergence. Unlike the steepest descent method, the search direction for two searching steps may happen to be parallel. The idea of conjugate gradient method is to keep memory of the previous direction and pick a next search direction orthogonal to all previous ones with adequate step size. However, calculation of the adequate step size may be time consuming. By increasing the value of each diagonal term in the Hessian matrix for Newton's method to avoid inverting a near-singular matrix, the Levenberg-Marquardt was developed. A nonnegative scaling factor for increasing the diagonal term is adjusted at each iteration. If an iteration gives insufficient reduction, this factor can be increased, and it blends more toward the simple gradient descent method. Whereas if reduction of the objective function is rapid, a smaller value can be used and the method becomes Newton's method. This method is well-known to have a fast convergence rate and also the convergence can be guaranteed [50].

In the present work, previously developed inverse radiation tools [51] for homogeneous turbulent single emitting gaseous media were extended based on the Levenberg-Marquardt method to deduce temperature and concentrations for gas mixtures from measurements of line-of-sight transmissivities. By assuming the pdf shape of temperature and species concentration fluctuations, time-averaged transmissivity and its rms spectrum were successfully related to the time-averaged and rms values of temperature and species concentration; this is the so-called forward calculation. Once these relationships were established, time-averaged and rms values of temperature and species concentration and turbulence scales can be retrieved from time-averaged transmissivities and their rms spectra; this is the so-called inverse calculation. To validate the model, synthetic turbulent fields for temperature and species concentration fluctuations were generated by a stochastic approach. Transmissivity spectra were generated along a line-of-sight for a mixture of $\mathrm{CO}_{2}, \mathrm{H}_{2} \mathrm{O}, \mathrm{CO}$ and inert $\mathrm{N}_{2}$ for instantaneous turbulent fields. The absorption coefficients, which are required to calculate transmissivity, were determined from HITEMP 2010, the high-temperature molecular spectroscopic database [52]. The database has been extensively tested against measured FTIR spectra of $\mathrm{CO}_{2}$ [53-55] and $\mathrm{H}_{2} \mathrm{O}$ [56, 57]. Good agreement between measured and calculated spectra was found. The synthetic transmissivities measurements were used as input data to retrieve scalars for turbulent gas mixtures.

\section{Turbulent transitivity calculation}

Our preliminary work [51] for a homogeneous turbulent gas medium is limited to a single combustion species. However, combustion products usually are mixtures of several gas species. In this study, the model is extended to retrieve time-averaged and rms values for temperature, species concentrations as well as the turbulence length scale for a gas mixture containing three major combustion products, i.e., $\mathrm{CO}_{2}, \mathrm{H}_{2} \mathrm{O}$ and $\mathrm{CO}$. Here we define a parameter vector

$$
\mathbf{z}=\left[T, x_{\mathrm{CO}_{2}}, x_{\mathrm{H}_{2} \mathrm{O}}, x_{\mathrm{CO}}\right]=\left[z_{1}, z_{2}, z_{3}, z_{4}\right]
$$

which is a set consisting of temperate and species concentrations of $\mathrm{CO}_{2}, \mathrm{H}_{2} \mathrm{O}$ and $\mathrm{CO}$. In order to retrieve the scalars and their turbulent moments, it is important to have an accurate forward calculation model that

can relate the measured lower-resolution time-averaged transmissivity $\left\langle\tau_{\eta c}\right\rangle$ and its variance $\left\langle\tau_{\eta c}^{\prime 2}\right\rangle$ to the time- 
averaged temperature and concentrations $\left\langle z_{j}\right\rangle(j=1,2,3,4)$ and their variances $\left\langle z_{j}^{\prime} z_{k}^{\prime}\right\rangle(j, k=1,2,3,4)$. Where a prime denotes a fluctuation about the local mean and angle brackets denote time-averaged quantities.

\subsection{LBL absorption coefficient}

For moderate fluctuations, the spectral absorption coefficient for the gas mixture can be approximated by a linearized function as

$$
\kappa_{\eta}(\mathbf{z}) \approx \kappa_{\eta}(\langle\mathbf{z}\rangle)+\sum_{j=1}^{4} \frac{\partial \kappa_{\eta}(\langle\mathbf{z}\rangle)}{\partial z_{j}} z_{j}^{\prime}
$$

where temperature and concentrations fluctuations $z_{j}^{\prime}$ are assumed to be Gaussian random variables with zero mean. Taking the average of Eq. (2) leads to

$$
\left\langle\kappa_{\eta}\right\rangle \approx \kappa_{\eta}(\langle\mathbf{z}\rangle)
$$

where $\langle\mathbf{z}\rangle$ is a set consisting of time-averaged temperature and species concentrations. This basically means the time-averaged absorption coefficient can be calculated from time-averaged temperature and species concentrations. The variance of the absorption coefficient is defined as the mean-square fluctuation of the absorption coefficient. Combining Eqs. (2) and (3), the variance of $\kappa_{\eta}$ can be deduced as

$$
\begin{aligned}
\left\langle\kappa_{\eta}^{\prime 2}\right\rangle & =\left\langle\left(\kappa_{\eta}-\left\langle\kappa_{\eta}\right\rangle\right)^{2}\right\rangle \\
& \approx\left\langle\left[\sum_{j=1}^{4} \frac{\partial\left\langle\kappa_{\eta}\right\rangle}{\partial z_{j}} z_{j}^{\prime}\right]^{2}\right\rangle \\
& =\sum_{j=1}^{4} \sum_{k=1}^{4} \frac{\partial\left\langle\kappa_{\eta}\right\rangle}{\partial z_{j}} \frac{\partial\left\langle\kappa_{\eta}\right\rangle}{\partial z_{k}}\left\langle z_{j}^{\prime} z_{k}^{\prime}\right\rangle
\end{aligned}
$$

Where $\left\langle\kappa_{\eta}^{\prime 2}\right\rangle$ is the variance of the absorption coefficient, $\left\langle z_{j}^{\prime} z_{k}^{\prime}\right\rangle(j, k=1,2,3,4)$ are variances of temperature and species concentrations, which can be variances of temperature $\left\langle T^{\prime 2}\right\rangle$, variances of species concentrations $\left\langle x_{j}^{\prime 2}\right\rangle$, covariances of temperature and concentrations $\left\langle T^{\prime} x_{j}^{\prime}\right\rangle$ and covariances of different species concentrations $\left\langle x_{j}^{\prime} x_{k}^{\prime}\right\rangle$.

\subsection{LBL trasmissivity}

Because of the approximately linear relation between absorption coefficients, temperature and species concentration, the absorption coefficient also varies temporally with a Gaussian distribution, which has a mean and variance of $\left\langle\kappa_{\eta}\right\rangle$ and $\left\langle\kappa_{\eta}^{\prime 2}\right\rangle$, respectively. It is known that the transmissivity is

$$
\tau_{\eta}=e^{-\int_{0}^{L} \kappa_{\eta}(s) \mathrm{d} s}
$$

Averaging over Eq. (5) yields the time-averaged LBL transmissivity

$$
\left\langle\tau_{\eta}\right\rangle=\left\langle e^{-\int_{0}^{L} \kappa_{\eta}(s) \mathrm{d} s}\right\rangle
$$

and the covariance of the LBL transmissivity at two arbitrary wavenumber locations

$$
\left\langle\tau_{\eta_{1}}^{\prime} \tau_{\eta_{2}}^{\prime}\right\rangle=\left\langle\left(\tau_{\eta_{1}}-\left\langle\tau_{\eta_{1}}\right\rangle\right)\left(\tau_{\eta_{2}}-\left\langle\tau_{\eta_{2}}\right\rangle\right)\right\rangle
$$


where $L$ is the length of the gas column. Detailed analysis for calculating these two terms can be found in [51] and the final expressions are presented here only. They are

$$
\left\langle\tau_{\eta}\right\rangle=e^{-\left\langle\kappa_{\eta}\right\rangle L} e^{\frac{1}{2}\left\langle\kappa_{\eta}^{\prime 2}\right\rangle L^{2} \int_{0}^{L} \int_{0}^{L} C_{s}\left(\left|s_{1}-s_{2}\right|\right) \mathrm{d} s_{1} \mathrm{~d} s_{2}}
$$

and

$$
\left\langle\tau_{\eta_{1}}^{\prime} \tau_{\eta_{2}}^{\prime}\right\rangle \approx\left\langle\tau_{\eta_{1}}\right\rangle\left\langle\tau_{\eta_{2}}\right\rangle \int_{0}^{L} \int_{0}^{L} C_{s}\left(\left|s_{1}-s_{2}\right|\right) \mathrm{d} s_{1} \mathrm{~d} s_{2} \sum_{j=1}^{4} \sum_{k=1}^{4} \frac{\partial\left\langle\kappa_{\eta_{1}}\right\rangle}{\partial z_{j}} \frac{\partial\left\langle\kappa_{\eta_{2}}\right\rangle}{\partial z_{k}}\left\langle z_{j}^{\prime} z_{k}^{\prime}\right\rangle
$$

where $C_{s}(r)=e^{-r / \Lambda}$ is the two-point/one-time correlation function for the turbulence field. Here $r$ is the distance between two arbitrary spatial locations $s_{1}$ and $s_{2}$, and $\Lambda$ is the turbulence integral length scale. Kritzstein and Soufiani [58] studied the TRI effect for different forms of the spatial correlation function and concluded that the contribution of turbulence on radiation is not very sensitive to the shape of the spatial correlation function. Therefore, in this study we only use exponential decay functions for spatial correlation functions. State-of-theart detectors that are in use to measure radiation from a turbulent gaseous medium are generally infrared array spectrometers, which are not fast enough to resolve turbulent fluctuations in the temporal domain. Therefore, for the theoretical investigation, we assume that the turbulent measurements that can be obtained are temporally independent and spatially correlated. Correspondingly, only spatial correlation functions are considered in the present study, and the turbulent length scale $\Lambda$ is retrieved simultaneously with temperature and species concentrations.

\subsection{Lower-resolution transmissivity}

Since transmissivity spectra can only be measured at a finite resolution by a spectrometer, the LBL spectral transmissivities have to be convolved with an pixel response function (PRF) to mimic the resolution of a spectrometer. After the transmissivity spectra are convolved with the $\operatorname{PRF} \Gamma(\eta)$, they become,

$$
\begin{aligned}
\left\langle\tau_{\eta c}\right\rangle & =\left\langle\int_{0}^{\infty} \tau_{\eta_{1}} \Gamma\left(\eta-\eta_{1}\right) \mathrm{d} \eta_{1}\right\rangle \\
& =\int_{0}^{\infty}\left\langle\tau_{\eta_{1}}\right\rangle \Gamma\left(\eta-\eta_{1}\right) \mathrm{d} \eta_{1}
\end{aligned}
$$

This implies that the convoluted time-averaged lower-resolution transmissivity equals the convolution of the time-averaged LBL transmissivity.

The variance of the convoluted transmissivity is

$$
\begin{aligned}
\left\langle\tau_{\eta c}^{\prime 2}\right\rangle & =\left\langle\left[\int_{0}^{\infty} \tau_{\eta_{1}} \Gamma\left(\eta-\eta_{1}\right) \mathrm{d} \eta_{1}-\int_{0}^{\infty}\left\langle\tau_{\eta_{1}}\right\rangle \Gamma\left(\eta-\eta_{1}\right) \mathrm{d} \eta_{1}\right]^{2}\right\rangle \\
& =\left\langle\left[\int_{0}^{\infty} \tau_{\eta_{1}}^{\prime} \Gamma\left(\eta-\eta_{1}\right) \mathrm{d} \eta_{1}\right]^{2}\right\rangle \\
& =\int_{0}^{\infty} \int_{0}^{\infty}\left\langle\tau_{\eta_{1}}^{\prime} \tau_{\eta_{2}}^{\prime}\right\rangle \Gamma\left(\eta-\eta_{1}\right) \Gamma\left(\eta-\eta_{2}\right) \mathrm{d} \eta_{1} \mathrm{~d} \eta_{2}
\end{aligned}
$$

where $\left\langle\tau_{\eta_{1}}\right\rangle$ and $\left\langle\tau_{\eta_{1}}^{\prime} \tau_{\eta_{2}}^{\prime}\right\rangle$ can be determined by Eqs. (8) and (9), respectively.

Up to this point, we have successfully related the measured time-averaged transmissivity $\left\langle\tau_{\eta c}\right\rangle$ and its variance $\left\langle\tau_{\eta c}^{\prime 2}\right\rangle$ to time-averaged temperature and concentrations $\left\langle z_{j}\right\rangle(j=1,2,3,4)$ and their variances $\left\langle z_{j}^{\prime} z_{k}^{\prime}\right\rangle$ $(j, k=1,2,3,4)$. Since $\left\langle z_{j}^{\prime} z_{k}^{\prime}\right\rangle=\left\langle z_{k}^{\prime} z_{j}^{\prime}\right\rangle$, there are 4 parameters for time-averaged temperature and species concentrations, 4 parameters for the variances of temperature and species concentrations, 3 parameters for the covariances of temperature and different species concentration and 3 parameters for the covariances of different 
species concentrations. Adding the turbulence length scale, in total there are 15 parameters to be retrieved for a turbulent mixture containing three species.

\section{Turbulent scalars inversion}

For the gas mixture, the transmissivity and its variance, Eqs. (10) and (11) can be written as

$$
\begin{aligned}
& f_{1 \eta}\left(\mathbf{z}_{1}, \mathbf{z}_{2}\right)=\left\langle\tau_{\eta c}\right\rangle \\
& f_{2 \eta}\left(\mathbf{z}_{1}, \mathbf{z}_{2}\right)=\left\langle\tau_{\eta c}^{\prime 2}\right\rangle
\end{aligned}
$$

Where $f_{1 \eta}$ and $f_{2 \eta}$ are nonlinear functions. Here we separate all unknown parameters into two parameter vectors, i.e.,

$$
\begin{aligned}
& \mathbf{z}_{1}=\left[\langle T\rangle,\left\langle x_{\mathrm{CO}_{2}}\right\rangle,\left\langle x_{\mathrm{H}_{2} \mathrm{O}}\right\rangle,\left\langle x_{\mathrm{CO}}\right\rangle\right] \\
& \mathbf{z}_{2}=\left[\left\langle T^{\prime 2}\right\rangle,\left\langle x_{\mathrm{CO}_{2}}^{\prime 2}\right\rangle,\left\langle x_{\mathrm{H}_{2} \mathrm{O}}^{\prime 2}\right\rangle,\left\langle x_{\mathrm{CO}}^{\prime 2}\right\rangle,\left\langle T^{\prime} x_{\mathrm{CO}_{2}}^{\prime}\right\rangle,\left\langle T^{\prime} x_{\mathrm{H}_{2} \mathrm{O}}^{\prime}\right\rangle,\left\langle T^{\prime} x_{\mathrm{CO}}^{\prime}\right\rangle,\left\langle x_{\mathrm{CO}_{2}}^{\prime} x_{\mathrm{H}_{2} \mathrm{O}}^{\prime}\right\rangle,\left\langle x_{\mathrm{CO}_{2}}^{\prime} x_{\mathrm{CO}}^{\prime}\right\rangle,\left\langle x_{\mathrm{H}_{2} \mathrm{O}}^{\prime} x_{\mathrm{CO}}^{\prime}\right\rangle, \Lambda\right]
\end{aligned}
$$

In principle, either of Eqs. (12) and (13) can be used to solve for all the parameters if one measures the timeaveraged transmissivity or the variance of transmissivity with a certain spectral resolution, obtaining enough discrete values at different wavenumbers. As discussed in [51], these two equations show different sensitivities to different parameters. For example, the time-averaged transmissivity is not sensitive to the intensity of turbulence fluctuations, but turbulent fluctuations always have significant effects on the fluctuation of transmissivities. In this study, time-averaged parameters were solved using Eq. (12), while variances, covariances and the turbulent length scale were retrieved from Eq. (13). Deducing $\mathbf{z}_{1}$ and $\mathbf{z}_{2}$ from Eqs. (12) or (13) requires deconvolution and makes these problems ill-posed and, therefore, these equations cannot be inverted directly to obtain all the parameters. Inverse procedures are required to solve them numerically. As was done for a single-species turbulent medium [51], the Levenberg-Marquardt is also employed in the present study. Details for the computational algorithm using the Levenberg-Marquardt method can be found in [51].

\section{Model validation}

\subsection{Synthetic turbulent measurements}

In the absence of measured spectra, in order to validate the model, "measured" spectra were synthesized through calculations from HITEMP 2010 for $\mathrm{CO}_{2}, \mathrm{H}_{2} \mathrm{O}$ and $\mathrm{CO}$. A stochastic approach is used to generate synthetic turbulent fields (fluctuations of temperature and species concentrations), as developed by Kritzstein and Soufiani [58] and adopted here, in which instantaneous temperature and species concentration fields are generated by Fourier transforming an assumed space-time correlation function. Without specifying the entire geometry of the system, turbulent scalar fields were created along a line-of-sight over a gas column of length $L$ and for the time interval $0 \leqslant t \leqslant t_{0}$. The scalar fields were assumed to be stationary, homogeneous stochastic processes, with a Gaussian probability density function. The reader is referred to [51, 58, 59] for more detail on the approach of numerically generating turbulent scalar fields. Temperature and species concentration fields were then generated by

$$
\mathbf{z}(s, t)=\mathbf{z}_{0}+\mathbf{z}^{\prime}(s, t)
$$

where $\mathbf{z}_{0}$ are the local mean values and $\mathbf{z}^{\prime}(s, t)$ are local instantaneous turbulent fluctuations, which have the following properties:

$$
\begin{gathered}
\left\langle z^{\prime}(s, t)\right\rangle=0 \\
\langle z(s, t) z(s+r, t+\tau)\rangle=\left\langle z^{\prime 2}\right\rangle C(r, \tau)
\end{gathered}
$$


The generated scalar field $z^{\prime}(s, t)$ is assumed to have a zero mean, a standard deviation of $\sqrt{\left\langle z^{2}\right\rangle}$ and to statistically satisfy a space-time correlation function:

$$
C(r, \tau)=C_{s}(r) C_{t}(\tau)
$$

where $C_{s}(r)=e^{-r / \Lambda}$ is the two-point/one-time correlation function and $C_{t}(\tau)=e^{-\tau / t_{e}}$ is the one-point/two-time correlation function, and $\Lambda$ and $t_{e}$ are the turbulence integral length and time scales, respectively.

In the present study, instantaneous scalar fields were created with an integral length scale of $\Lambda$ for a gas medium of length $L$, and with an integral time scale of $t_{e}$ for a total time interval of $t$. Instantaneous turbulence fields were generated so that temperature and species concentration have certain percentages of fluctuations around the mean values. Temperature and species concentration fields were created for 100 spatial points along the gas column of length $L$ and for 1000 time realizations in $1 \mathrm{~s}$. The integral time scale was set to $0.1 \mathrm{~ms}$, which makes a temporally independent turbulence field. Once temperature and species concentration fields along a line-of-sight were obtained, instantaneous LBL spectral transmissvities $\tau_{\eta}(t)$ can be generated, after which the LBL spectral transmissivities are convolved with a PRF create instantaneous lower-resolution transmissivities $\tau_{\eta c}(t)$. Without convolution the spectrum contains information for each individual transition line and contains more spectral information. However, spectral data are based on quantum mechanical calculations together with experimental measurements, and are subject to some degree of theoretical uncertainty. In an experiment data are smoothed out by convolving the line-by-line spectrum with a PRF. After convolution smoother averaged shapes are obtained, but very coarse resolution would smoothed out variations of the spectral transmissivity with wavenumber and some of the spectral information is permanently lost. Here we simulate how a spectrum is measured by a detector. If important spectral information is lost due to the convolution, that simply means the measurement resolution is not fine enough. In the present study, LBL spectral transmissivities are convolved with an PRF (the PRF used here remains the same as in [51]) with medium nominal resolution of $4 \mathrm{~cm}^{-1}$ to create instantaneous lower-resolution transmissivity spectra based on the generated turbulent scalars filed.

The time-averaged transmissivity $\left\langle\tau_{\eta c}\right\rangle$ and its variance $\left\langle\tau_{\eta c}^{\prime 2}\right\rangle$ can be obtained by conducting a stochastic analysis over the instantaneous transmissivity [51]. These values were used as "actual" measurement data in Eqs. (12) and (13) to retrieve parameters $\mathbf{z}_{1}$ and $\mathbf{z}_{2}$. For the current study, we did not perturb our synthetic data by random noise because: (1) when generating time-averaged synthetic transmissivity data, the noise will be simply smoothed out when calculating the average; (2) although adding random noise to the instantaneous synthetic transmissivity data will significantly alter the rms synthetic transmissivity spectrum, techniques may be applied to filter random noise from turbulent signals [60, 61] or distinguish uncorrelated measurement noise from correlated turbulent fluctuations [62]. However, this is beyond the scope of the current study.

\subsection{Results and discussion}

\subsubsection{Spectral bands selection}

Radiation spectra for the three species contain several absorption bands, which are at different spectral locations for different species and vary with different conditions. In order to retrieve retrieve the turbulent scalars and their moments accurately and efficiently, it is very important to choose appropriate spectral bands to conduct the inverse calculations. Figure 1 shows the spectral absorption coefficients for the three species (5\% $\mathrm{CO}_{2}, 20 \% \mathrm{H}_{2} \mathrm{O}$ and $5 \% \mathrm{CO}$ ) at 1 bar and a temperature of $1500 \mathrm{~K}$. These conditions were chosen to represent typical physical conditions of laboratory methane flames [63, 64] for a location where incomplete combustion has taken place. In this part of the spectrum, $\mathrm{CO}_{2}$ has two strong bands and $\mathrm{CO}$ has one strong band and one weak band. For $\mathrm{H}_{2} \mathrm{O}$, the bands are wider and spread out over the spectrum. Ideally, choosing a part of the spectrum where all three species overlap may retrieve all the parameters simultaneously. For example, all three species have overlapping bands in the $1800-2500 \mathrm{~cm}^{-1}$ spectral interval (marked as spectral interval I for later reference), which can be explored for inversion. As shown in Fig. 1 $\mathrm{CO}_{2}$ and $\mathrm{H}_{2} \mathrm{O}$ also have overlapping absorption bands in the $3200-3800 \mathrm{~cm}^{-1}$ spectral interval (spectral interval II), which may be used to retrieve turbulent scalars. However, only parameters for $\mathrm{CO}_{2}$ and $\mathrm{H}_{2} \mathrm{O}$ can be retrieved simultaneously; $\mathrm{CO}$ parameters can never be retrieved from this part of the spectrum. As also shown in Fig. 11 there is a weak CO absorption band within the $4000 \mathrm{~cm}^{-1}-4400 \mathrm{~cm}^{-1}$ spectral interval, which overlaps with $\mathrm{H}_{2} \mathrm{O}$, and this region can be used 


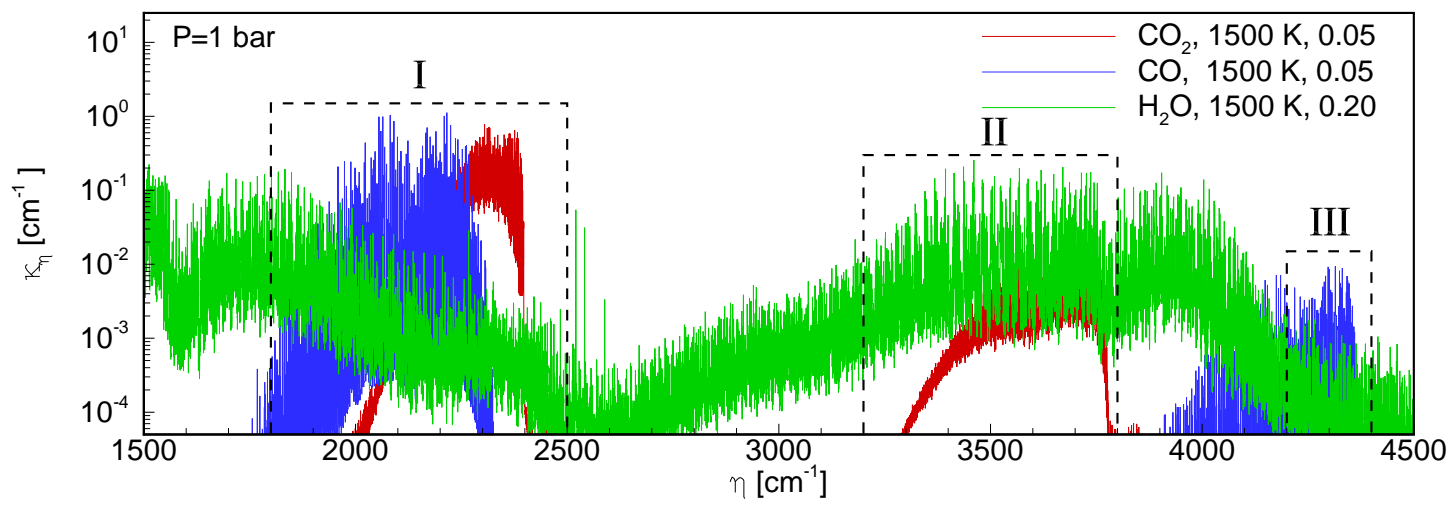

Figure 1: Spectral absorption coefficient for three combustion species: $\mathrm{CO}_{2}, \mathrm{H}_{2} \mathrm{O}$ and $\mathrm{CO}$

to retrieve parameters for $\mathrm{CO}$. However, the band is weak and the spectrum for $\mathrm{H}_{2} \mathrm{O}$ is dominating over $\mathrm{CO}$. This makes it difficult to retrieve CO parameters in most practical situations. Our tests indicate that using the $4200 \mathrm{~cm}^{-1}-4400 \mathrm{~cm}^{-1}$ spectral interval (spectral interval III) can retrieve CO parameters more accurately than using the $4000 \mathrm{~cm}^{-1}-4400 \mathrm{~cm}^{-1}$ spectral interval. In addition, the covariance between two different species cannot be retrieved if there is no overlap of the absorption bands of the two species. Therefore, the capability of each band to retrieve turbulent scalars must be investigated at different conditions.

To illustrate typical behaviours, we consider a mean temperature of $1500 \mathrm{~K}$ and mean concentrations for $\mathrm{CO}_{2}, \mathrm{H}_{2} \mathrm{O}$ and $\mathrm{CO}$ of 5\%, 20\%, 5\%, respectively. Both temperature and species concentrations have $10 \%$ turbulent fluctuations around their mean values. The total pressure is 1 bar and the gas column length is 100 $\mathrm{cm}$, while the turbulence integral length scale is $10 \mathrm{~cm}(\Lambda=0.1 \mathrm{~L})$. To understand the type of information that each parameter contributes to the "measured" spectra under these conditions, one must examine the sensitivity of the spectra to the parameters to be retrieved. In Eq. (9), let

$$
\theta(\Lambda)=\int_{0}^{L} \int_{0}^{L} C_{s}\left(\left|s_{1}-s_{2}\right|\right) \mathrm{d} s_{1} \mathrm{~d} s_{2}
$$

Then Eq. (11) can be rewritten as

$$
\begin{aligned}
\left\langle\tau_{\eta c}^{\prime 2}\right\rangle & \approx \theta(\Lambda) \sum_{j=1}^{4} \sum_{k=1}^{4} \int_{0}^{\infty} \int_{0}^{\infty}\left\langle\tau_{\eta_{1}}\right\rangle\left\langle\tau_{\eta_{2}}\right\rangle \frac{\partial\left\langle\kappa_{\eta_{1}}\right\rangle}{\partial z_{j}} \frac{\partial\left\langle\kappa_{\eta_{2}}\right\rangle}{\partial z_{k}} \Gamma\left(\eta-\eta_{1}\right) \Gamma\left(\eta-\eta_{2}\right) \mathrm{d} \eta_{1} \mathrm{~d} \eta_{2}\left\langle z_{j}^{\prime} z_{k}^{\prime}\right\rangle \\
& =\sum_{j=1}^{4} \sum_{k=1}^{4} W_{\eta, j k}\left\langle z_{j}^{\prime} z_{k}^{\prime}\right\rangle
\end{aligned}
$$

where

$$
W_{\eta, j k}=\frac{\partial\left\langle\tau_{\eta c}^{\prime 2}\right\rangle}{\partial\left\langle z_{j}^{\prime} z_{k}^{\prime}\right\rangle} \approx \theta(\Lambda) \int_{0}^{\infty} \int_{0}^{\infty}\left\langle\tau_{\eta_{1}}\right\rangle\left\langle\tau_{\eta_{2}}\right\rangle \frac{\partial\left\langle\kappa_{\eta_{1}}\right\rangle}{\partial z_{j}} \frac{\partial\left\langle\kappa_{\eta_{2}}\right\rangle}{\partial z_{k}} \Gamma\left(\eta-\eta_{1}\right) \Gamma\left(\eta-\eta_{2}\right) \mathrm{d} \eta_{1} \mathrm{~d} \eta_{2}
$$

is the sensitivity coefficient with respect to the parameter $\left\langle z_{j}^{\prime} z_{k}^{\prime}\right\rangle$, which determines the sensitivity of the spectral signal to the parameters to be retrieved. However, direct comparison of the coefficients is not appropriate since they have different units and usually differ by several orders of magnitude. Proper comparison may be obtained using the relative sensitivity coefficients defined as [65],

$$
\bar{W}_{\eta, j k}=\left\langle z_{j}^{\prime} z_{k}^{\prime}\right\rangle \frac{\partial\left\langle\tau_{\eta c}^{\prime 2}\right\rangle}{\partial\left\langle z_{j}^{\prime} z_{k}^{\prime}\right\rangle}
$$



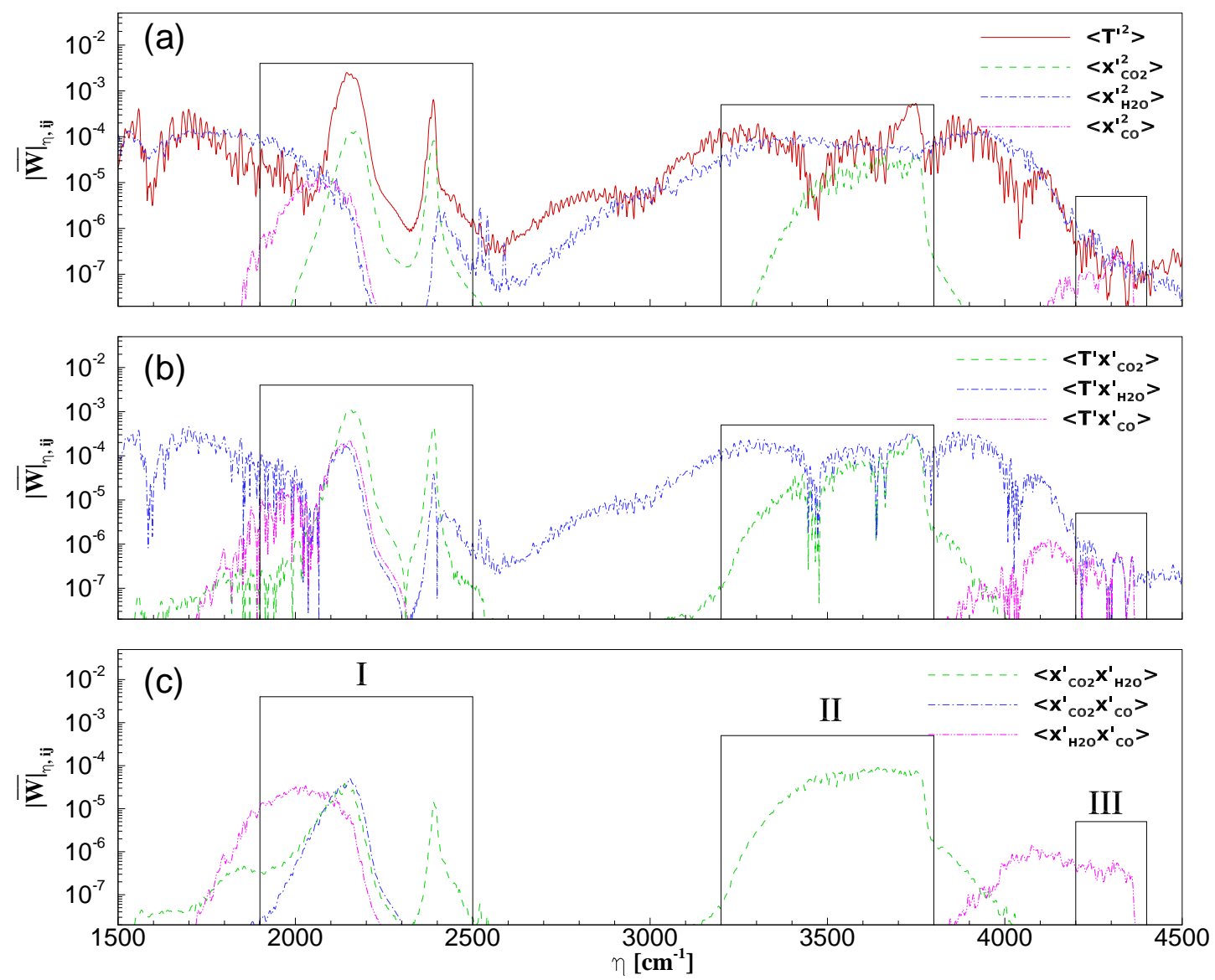

Figure 2: Relative sensitivity coefficients of: (a) variance of temperature, species concentrations, (b) covariance of temperature and species concentrations and (c) covariance of different species concentrations for a gas mixture of $5 \% \mathrm{CO}_{2}+20 \% \mathrm{H}_{2} \mathrm{O}+5 \% \mathrm{CO}, 1500 \mathrm{~K}, P=1$ bar, $L=100 \mathrm{~cm}, \sqrt{\left\langle z^{\prime 2}\right\rangle} /\langle z\rangle=10 \%$ and $\Lambda / L=0.1$

These tend to be different from one parameter to another and also different from one spectral band to another spectral band. It is very important to select spectral bands, which give well-balanced accuracy for all parameters of interest.

Figure 2 shows absolute values of relative sensitivity coefficients. In spectral interval I, as shown in the figure, the contributions from the variance of temperature and covariance of temperature and $\mathrm{CO}_{2}$ concentration dominate and are orders of magnitude higher than that of other parameters. Thus one cannot expect all parameters to be retrieved having well-balanced accuracy using this part of the spectrum. Our extensive tests indicate that only time-averaged values for the gas mixture may be retrieved accurately. As shown in Fig. 1, $\mathrm{CO}_{2}$ and $\mathrm{H}_{2} \mathrm{O}$ have overlapping absorption bands in spectral interval II. For the given conditions the relative sensitivity coefficients for all other parameters related to these two species show well balanced values in this spectral interval, i.e., they are of the same order of magnitude, as shown in Fig. 2. Using this part of the spectrum, all parameters for $\mathrm{CO}_{2}$ and $\mathrm{H}_{2} \mathrm{O}$ can be retrieved simultaneously. As discussed, time-averaged temperatures and species concentrations for all three species may be retrieved from spectral interval I and all parameters related to $\mathrm{CO}_{2}$ and $\mathrm{H}_{2} \mathrm{O}$ may be retrieved from spectral interval II. There are 4 remaining parameters related to $\mathrm{CO}$ that may need to be retrieved. While CO has one major absorption band in spectral interval I, as stated before, only the time-averaged $\mathrm{CO}$ concentration can be retrieved simultaneously with other time-averaged parameters from this spectral range. As indicated in [51], local linearization of the absorption coefficient with temperature and 
gas concentrations is not a very good approximation for a spectral interval where the absorption coefficient is very large, and Eq. (9) is only valid if the covariance of the turbulent optical thickness is small. Spectral interval I contains the $\mathrm{CO}_{2} 4.3 \mu \mathrm{m}$ band, which is very strong. Even for a gas containing only $\mathrm{CO}_{2}$, using this band cannot retrieve rms values for temperature and concentration very well. But $\mathrm{CO}$ has another absorption band in spectral interval III, as shown in Fig. 2 which makes it possible to retrieve the remaining parameters from this part of the spectrum if enough $\mathrm{CO}$ is present. However, there are no $\mathrm{CO}_{2}$ bands in this part of the spectrum and, consequently, $\left\langle x_{\mathrm{CO}_{2}}^{\prime} x_{\mathrm{CO}}^{\prime}\right\rangle$ cannot be retrieved. Therefore instead of retrieving all parameters simultaneously from spectral interval I, the remaining 3 parameters, i.e., $\left\langle x_{\mathrm{CO}}^{\prime 2}\right\rangle,\left\langle T^{\prime} x_{\mathrm{CO}}^{\prime}\right\rangle$, and $\left\langle x_{\mathrm{H}_{2} \mathrm{O}}^{\prime} x_{\mathrm{CO}}^{\prime}\right\rangle$ can be retrieved from spectral interval III after all other parameters have been found from spectral intervals I and II. To summarize, the following conclusions may be drawn:

- All parameters for the three species can be retrieved simultaneously from spectral interval I, however, only mean values may be retrieved relatively accurately. If only mean values are of interest, spectral interval I may be used to retrieve these values.

- All the parameters related to $\mathrm{CO}_{2}$ and $\mathrm{H}_{2} \mathrm{O}$ and turbulent length scale can be retrieved simultaneously from spectral interval II. Therefore, if there is no $\mathrm{CO}$ presents in the mixture or parameters only related to $\mathrm{CO}_{2}$ and $\mathrm{H}_{2} \mathrm{O}$ are of interest, one may retrieve these values simultaneously from spectral interval II.

- For a gas mixture of $\mathrm{CO}_{2}, \mathrm{H}_{2} \mathrm{O}$ and $\mathrm{CO}$, with mean parameters retrieved from spectral interval I and other parameters retrieved from spectral interval II, the parameters related to $\mathrm{CO}$ except $\left\langle x_{\mathrm{CO}_{2}}^{\prime} x_{\mathrm{CO}}^{\prime}\right\rangle$ may be retrieved from spectral interval III.

Analyzing the sensitivity of the spectrum to the parameters to be retrieved can give more insight on how to choose appropriate spectral intervals to conduct inverse calculations. However, the relative sensitivity coefficient for each parameter, as shown in Fig. 2 can only be obtained when the physical conditions are known. That is normally not possible because the objective of the inverse calculations is to retrieve these physical conditions. However, tests indicate that while the relative sensitivity coefficient is different for different physical conditions, the patterns and orders of magnitude remain similar over fairly large ranges of conditions. This implies that choosing bands from this particular case may hold for a large range of conditions. To investigate the capability of retrieving turbulent scalars from turbulent transmission "measurements" from the three identified spectral ranges, cases were tested with different combinations of species abundance, different temperatures, different turbulent intensities, different turbulent length scale and different turbulent length scales.

\subsubsection{Species concentrations}

Mixture of $5 \% \mathrm{CO}_{2}, 20 \% \mathrm{H}_{2} \mathrm{O}$ and $5 \% \mathrm{CO}$ with $\mathrm{N}_{2}$

The first case is for the conditions given in Section 4.2.1. Table 1 shows the "retrieved" parameters of the mixture from the three identified spectral intervals compared to the "actual values," which are directly calculated from the generated turbulence fields by conducting a stochastic analysis and are used as benchmark for retrieved values from inverse calculations. In the table, time-averaged parameters were retrieved from spectral interval I; all parameters related to $\mathrm{CO}_{2}$ and $\mathrm{H}_{2} \mathrm{O}$ (mean and rms values) and the turbulence length scale were retrieved from spectral interval II and other parameters related to $\mathrm{CO}$ (except $\left.\left\langle x_{\mathrm{CO}_{2}}^{\prime} x_{\mathrm{CO}}^{\prime}\right\rangle\right)$ were retrieved from spectral interval III after determining all other parameters. Retrieved time-averaged results are fairly accurate: differences from actual parameter values are less than or about $5 \%$. The retrieved rms value for $\mathrm{CO}_{2}$ concentration and covariance of $\mathrm{CO}_{2}$ and $\mathrm{H}_{2} \mathrm{O}$ concentration from spectral interval II, as well as rms value for $\mathrm{CO}$ concentration from spectral interval III, show relatively larger differences compared to the "actual values." The relative sensitivity coefficients for these parameters are relatively small, indicating that they contribute less to the variance of transmissivities, as shown in Fig 2. Therefore, they are relatively less sensitive to the variance of the transmissivity spectrum.

In Fig. 3 actual time-averaged transmissivities and their rms spectra are compared with the spectra calculated from forward calculations from Eqs. (10) and 111) with the actual parameter values listed in Table 1 , and are also compared to the spectra calculated with retrieved parameter values given in Tables 1 The retrieved spectra were calculated using the time-averaged temperature and concentrations from spectral interval I, and 
Table 1: Inverse calculation results for conditions: $5 \% \mathrm{CO}_{2}+20 \% \mathrm{H}_{2} \mathrm{O}+5 \% \mathrm{CO}, 1500 \mathrm{~K}, P=1$ bar, $L=100 \mathrm{~cm}$, $\sqrt{\left\langle z^{\prime 2}\right\rangle} /\langle z\rangle=10 \%$ and $\Lambda / L=0.1$

\begin{tabular}{|c|c|c|c|c|c|c|}
\hline & retrieved parameters & $\langle T\rangle(\mathrm{K})$ & $\left\langle x_{\mathrm{CO}_{2}}\right\rangle$ & $\left\langle x_{\mathrm{H}_{2}} \mathrm{O}\right\rangle$ & $\left\langle x_{\mathrm{CO}}\right\rangle$ & $\sqrt{\left\langle T^{\prime 2}\right\rangle}(\mathrm{K})$ \\
\hline \multirow{3}{*}{ I } & actual values & 1495 & 0.0498 & 0.1994 & 0.0498 & 150.8 \\
\hline & retrieved & 1514 & 0.0510 & 0.1985 & 0.0524 & - \\
\hline & error(\%) & 1.24 & 2.41 & -0.41 & 5.17 & - \\
\hline \multirow{4}{*}{ II } & retrieved & 1487 & 0.0492 & 0.1999 & - & 151.7 \\
\hline & error $(\%)$ & -0.52 & -1.33 & 0.26 & - & 0.57 \\
\hline & retrieved parameters & $\sqrt{\left\langle x_{\mathrm{CO}_{2}}^{\prime 2}\right\rangle}$ & $\sqrt{\left\langle x_{\mathrm{H}_{2} \mathrm{O}}^{\prime 2}\right\rangle}$ & $\left\langle T^{\prime} x_{\mathrm{CO}_{2}}^{\prime}\right\rangle(\mathrm{K})$ & $\left\langle T^{\prime} x_{\mathrm{H}_{2} \mathrm{O}}^{\prime}\right\rangle(\mathrm{K})$ & $\left\langle x_{\mathrm{CO}_{2}}^{\prime} x_{\mathrm{H}_{2} \mathrm{O}}^{\prime}\right\rangle$ \\
\hline & actual values & 0.0050 & 0.0201 & 0.7584 & 3.0335 & $1.01 \mathrm{E}-04$ \\
\hline \multirow{4}{*}{ II } & retrieved & 0.0042 & 0.0184 & 0.8146 & 2.9368 & $1.20 \mathrm{E}-04$ \\
\hline & error $(\%)$ & -17.06 & -8.47 & 7.41 & -3.18 & 18.30 \\
\hline & retrieved parameters & $\sqrt{\left\langle x_{\mathrm{CO}}^{\prime 2}\right\rangle}$ & $\left\langle T^{\prime} x_{\mathrm{CO}}^{\prime}\right\rangle(\mathrm{K})$ & $\left\langle x_{\mathrm{CO}_{2}}^{\prime} x_{\mathrm{CO}}^{\prime}\right\rangle$ & $\left\langle x_{\mathrm{H}_{2} \mathrm{O}}^{\prime} x_{\mathrm{CO}}^{\prime}\right\rangle$ & $\Lambda / L$ \\
\hline & actual values & 0.0050 & 0.7584 & - & $1.01 \mathrm{E}-04$ & 0.100 \\
\hline \multirow{2}{*}{ II } & retrieved & - & - & - & - & 0.102 \\
\hline & error $(\%)$ & - & - & - & - & 2.38 \\
\hline \multirow{2}{*}{ III } & retrieved & 0.0062 & 0.8433 & - & $8.99 \mathrm{E}-05$ & - \\
\hline & error $(\%)$ & 22.94 & 11.20 & - & -11.11 & - \\
\hline
\end{tabular}
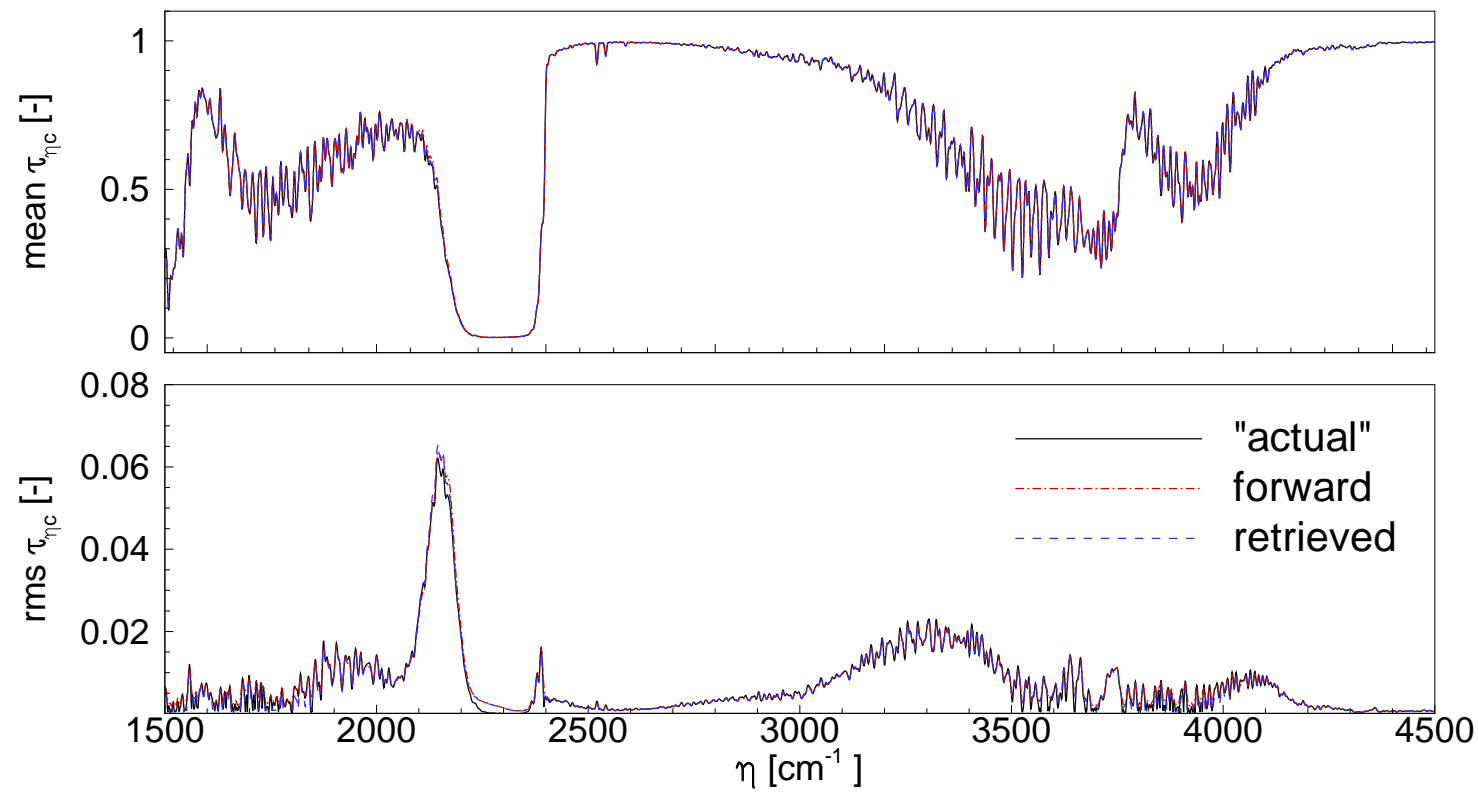

Figure 3: Comparison of retrieved transmissivity and its rms spectra with the "actual" spectra and forward spectra calculated by the actual turbulent scalars for a gas mixture of $5 \% \mathrm{CO}_{2}+20 \% \mathrm{H}_{2} \mathrm{O}+5 \% \mathrm{CO}$

all other parameters from spectral intervals II and III. For these time-averaged transmissivities and their rms spectra, retrieved and forward spectra perfectly overlap with the "actual" spectra. Although "retrieved" and "forward" rms spectra show discrepancies with the "actual" rms spectra at smaller rms values, the spectral peaks and most parts of the "actual" rms spectra bands are well captured by the "retrieved" and "forward" rms spectra. This shows that the forward calculations to predict lower-resolution time-averaged transmissivities and their rms values are accurate enough and the resulting inverse radiation model provides a reliable tool for retrieving mean temperature, concentrations, their rms values and turbulent length scale from synthetic turbulent transmissivity measurements. 
Table 2: Inverse calculation results for conditions: $20 \% \mathrm{CO}_{2}+5 \% \mathrm{H}_{2} \mathrm{O}+5 \% \mathrm{CO}, 1500 \mathrm{~K}, P=1$ bar, $L=100 \mathrm{~cm}$, $\sqrt{\left\langle z^{\prime 2}\right\rangle} /\langle z\rangle=10 \%$ and $\Lambda / L=0.1$

\begin{tabular}{ccccccc}
\hline & retrieved parameters & $\langle T\rangle(\mathrm{K})$ & $\left\langle x_{\mathrm{CO}_{2}}\right\rangle$ & $\left\langle x_{\mathrm{H}_{2} \mathrm{O}}\right\rangle$ & $\left\langle x_{\mathrm{CO}}\right\rangle$ & $\sqrt{\left\langle T^{\prime 2}\right\rangle}(\mathrm{K})$ \\
\hline \multirow{4}{*}{$\mathrm{I}$} & actual values & 1495 & 0.1994 & 0.0498 & 0.0498 & 150.8 \\
& retrieved & 1524 & 0.2085 & 0.0500 & 0.0509 & - \\
& error(\%) & 1.91 & 4.61 & 0.29 & 2.20 & - \\
$\mathrm{II}$ & retrieved & 1502 & 0.1985 & 0.0505 & - & 148.9 \\
& error(\%) & 0.48 & -0.45 & 1.26 & - & -1.26 \\
\hline \multirow{2}{*}{} & retrieved parameters & $\sqrt{\left\langle x_{\mathrm{CO}}^{\prime 2}\right\rangle}$ & $\sqrt{\left\langle x_{\mathrm{H}_{2} \mathrm{O}}^{\prime 2}\right\rangle}$ & $\left\langle T^{\prime} x_{\mathrm{CO}_{2}}^{\prime}\right\rangle(\mathrm{K})$ & $\left\langle T^{\prime} x_{\mathrm{H}_{2} \mathrm{O}}^{\prime}\right\rangle(\mathrm{K})$ & $\left\langle x_{\mathrm{CO}_{2}}^{\prime} x_{\mathrm{H}_{2} \mathrm{O}}^{\prime}\right\rangle$ \\
\hline \multirow{4}{*}{$\mathrm{II}$} & actual values & 0.0201 & 0.0050 & 3.0335 & 0.7584 & $1.01 \mathrm{E}-04$ \\
& retrieved & 0.0205 & 0.0051 & 3.0845 & 0.7732 & $1.03 \mathrm{E}-04$ \\
& error(\%) & 2.16 & 1.87 & 1.68 & 1.96 & 2.10 \\
\hline \multirow{2}{*}{} & retrieved parameters & $\sqrt{\left\langle x_{\mathrm{CO}}^{\prime 2}\right\rangle}$ & $\left\langle T^{\prime} x_{\mathrm{CO}}^{\prime}\right\rangle(\mathrm{K})$ & $\left\langle x_{\mathrm{CO}_{2}}^{\prime} x_{\mathrm{CO}}^{\prime}\right\rangle$ & $\left\langle x_{\mathrm{H}_{2} \mathrm{O}}^{\prime} x_{\mathrm{CO}}^{\prime}\right\rangle$ & $\Lambda / L$ \\
\hline \multirow{2}{*}{$\mathrm{II}$} & actual values & 0.0050 & 0.7584 & - & $2.53 \mathrm{E}-05$ & 0.100 \\
& retrieved & - & - & - & - & 0.099 \\
& error(\%) & - & - & - & - & -1.32 \\
$\mathrm{III}$ & retrieved & 0.0047 & 0.7536 & - & $1.83 \mathrm{E}-05$ & - \\
& error(\%) & -5.68 & -0.63 & - & -27.45 & - \\
\hline
\end{tabular}

Mixture of $20 \% \mathrm{CO}_{2}, 5 \% \mathrm{H}_{2} \mathrm{O}$ and $5 \% \mathrm{CO}$ with $\mathrm{N}_{2}$

$\mathrm{CO}_{2}$ has relatively stronger absorption, especially across the $\mathrm{CO}_{2} 4.3 \mu \mathrm{m}$ band. Therefore, absorption from $\mathrm{CO}_{2}$ will dominate over the other two species in spectral interval I. On the other hand, we invoke the assumption that the spectral absorption coefficient is a linear function over the temperature range of $\langle T\rangle \pm T_{\max }^{\prime}$ and species concentration range of $\langle x\rangle \pm x_{\max }^{\prime}$ in the forward calculation, as given by Eq. (2). For the $\mathrm{CO}_{2} 4.3 \mu \mathrm{m}$ band, the spectral absorption coefficient is slightly nonlinear over the temperature range $1500 \pm 150 \mathrm{~K}$. Compared to other spectral bands, the $\mathrm{CO}_{2} 4.3 \mu \mathrm{m}$ band is so strong that slight nonlinearity may cause large error for larger optical thickness $\left(\left\langle\kappa_{\eta}\right\rangle L\right)$. An artificial case was created to test the performance of the proposed method for a gas mixture, which contains more $\mathrm{CO}_{2}$ than the other two species. In this test the mean temperature $T_{0}$ of the gas mixture is $1500 \mathrm{~K}$ and the mean concentration for $\mathrm{CO}_{2}$ is $20 \%$, while mean concentrations for $\mathrm{H}_{2} \mathrm{O}$ and $\mathrm{CO}$ are both 5\%. Again, temperature and species concentrations are assumed to have 10\% fluctuations around their mean values. Other conditions remain the same as in the previous case.

Although the values for the relative sensitivity coefficients are different from the previous case, the pattern and orders of magnitude are similar. The inverse results are summarized in Table 2 Figure 4 shows the comparison of retrieved transmissivity and their rms spectra with the "actual" spectra and spectra calculated from the forward calculation for the gas mixture. Agreement is very good, except the spectral location where the $\mathrm{CO}_{2} 4.3 \mu \mathrm{m}$ band is.

Mixture of $5 \% \mathrm{CO}_{2}, 5 \% \mathrm{H}_{2} \mathrm{O}$ and $20 \% \mathrm{CO}$ with $\mathrm{N}_{2}$

This case considers a gas mixture dominated by $\mathrm{CO}$, with mean concentration for $\mathrm{CO}$ of $20 \%$ and mean concentrations of the other two species both at 5\%. All other conditions remain the same as in the previous case. Again, turbulent scalars were retrieved following the same strategy as for the two previous cases. All retrieved parameters are given in Table 3. Figure 5 shows the comparison of retrieved transmissivities and their rms spectra with the "actual" spectra and spectra calculated from the forward calculation for the gas mixture; again, "actual" rms spectra are well captured by the retrieved and forward rms spectra.

\subsubsection{Temperatures, turbulent intensities, turbulence length scale}

For a $100 \mathrm{~cm}$ long gas mixture column of $5 \% \mathrm{CO}_{2}+20 \% \mathrm{H}_{2} \mathrm{O}+5 \% \mathrm{CO}$ with $\mathrm{N}_{2}$ at atmospheric pressure ( $1 \mathrm{bar}$ ), test cases were generated for mean temperatures of $1000 \mathrm{~K}$ and $2000 \mathrm{~K}$, turbulent fluctuations of $5 \%$ and $15 \%$, and a turbulence integral length scale $\Lambda / L=0.2$ to show the capability of the proposed methods for retrieving turbulent scalars. Again, $\langle T\rangle,\left\langle x_{\mathrm{CO}_{2}}\right\rangle,\left\langle x_{\mathrm{H}_{2} \mathrm{O}}\right\rangle$ and $\left\langle x_{\mathrm{CO}}\right\rangle$ are retrieved from spectral interval I; $\left\langle T^{\prime 2}\right\rangle$, 

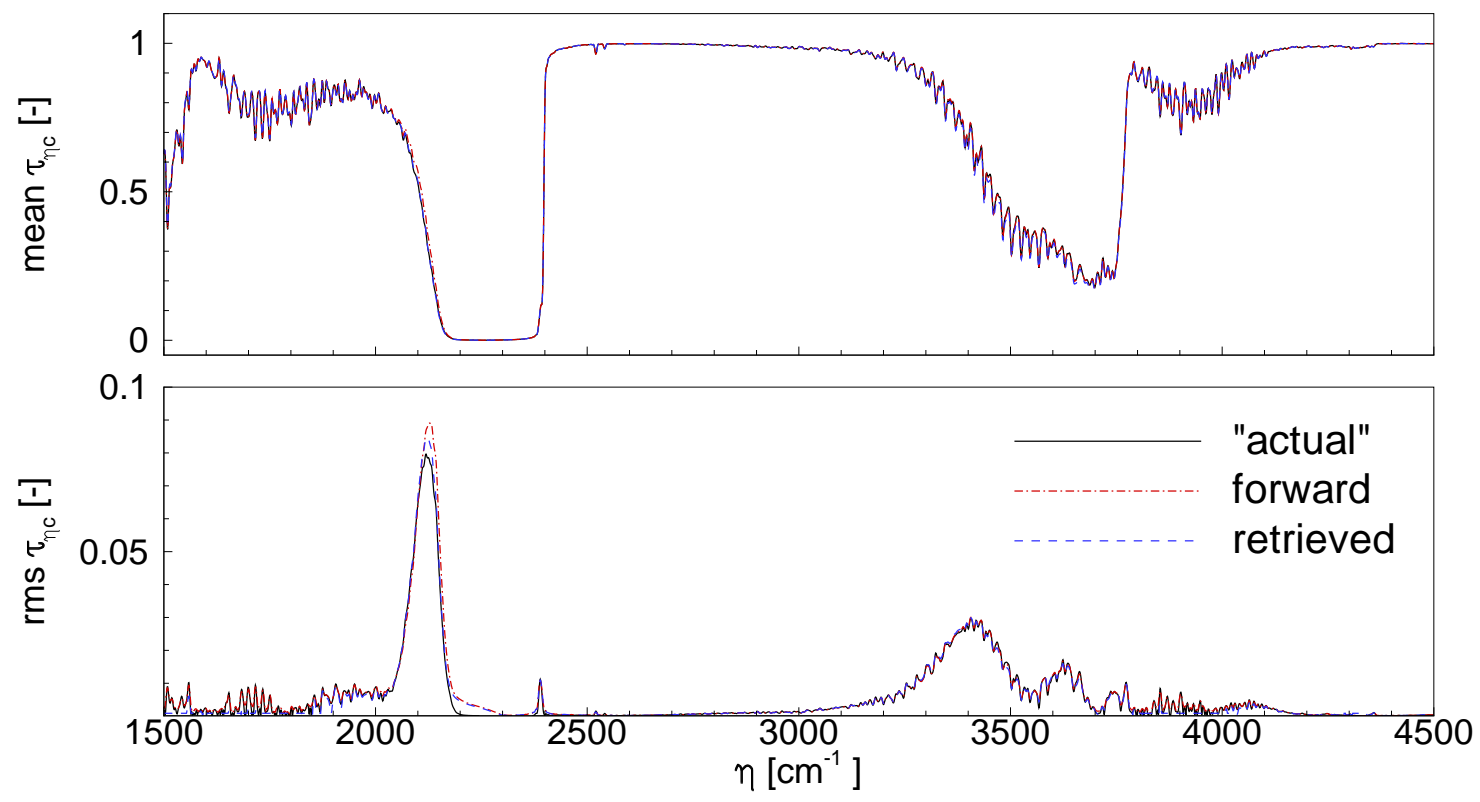

Figure 4: Comparison of retrieved transmissivity and its rms spectra with the "actual" spectra and forward spectra calculated by the actual turbulent scalars for a gas mixture of $20 \% \mathrm{CO}_{2}+5 \% \mathrm{H}_{2} \mathrm{O}+5 \% \mathrm{CO}$

Table 3: Inverse calculation results for conditions: $5 \% \mathrm{CO}_{2}+5 \% \mathrm{H}_{2} \mathrm{O}+20 \% \mathrm{CO}, 1500 \mathrm{~K}, P=1 \mathrm{bar}, L=100 \mathrm{~cm}$, $\sqrt{\left\langle z^{\prime 2}\right\rangle} /\langle z\rangle=10 \%$ and $\Lambda / L=0.1$

\begin{tabular}{|c|c|c|c|c|c|c|}
\hline & retrieved parameters & $\langle T\rangle(\mathrm{K})$ & $\left\langle x_{\mathrm{CO}_{2}}\right\rangle$ & $\left\langle x_{\mathrm{H}_{2} \mathrm{O}}\right\rangle$ & $\left\langle x_{\mathrm{CO}}\right\rangle$ & $\sqrt{\left\langle T^{\prime 2}\right\rangle}(\mathrm{K})$ \\
\hline \multirow{3}{*}{ I } & actual values & 1495 & 0.0498 & 0.0498 & 0.1994 & 150.8 \\
\hline & retrieved & 1513 & 0.0515 & 0.0497213464 & 0.2050 & - \\
\hline & error $(\%)$ & 1.22 & 3.26 & -0.23 & 2.83 & - \\
\hline \multirow{3}{*}{ II } & retrieved & 1492 & 0.0494 & 0.0500 & - & 151.4 \\
\hline & error $(\%)$ & -0.19 & -0.79 & 0.25 & - & 0.38 \\
\hline & retrieved parameters & $\sqrt{\left\langle x_{\mathrm{CO}_{2}}^{\prime 2}\right\rangle}$ & $\sqrt{\left\langle x_{\mathrm{H}_{2} \mathrm{O}}^{\prime 2}\right\rangle}$ & $\left\langle T^{\prime} x_{\mathrm{CO}_{2}}^{\prime}\right\rangle(\mathrm{K})$ & $\left\langle T^{\prime} x_{\mathrm{H}_{2} \mathrm{O}}^{\prime}\right\rangle(\mathrm{K})$ & $\left\langle x_{\mathrm{CO}_{2}}^{\prime} x_{\mathrm{H}_{2} \mathrm{O}}^{\prime}\right\rangle$ \\
\hline \multirow{5}{*}{ II } & actual values & 0.0050 & 0.0050 & 0.7584 & 0.7584 & $2.53 \mathrm{E}-05$ \\
\hline & retrieved & 0.0049 & 0.0046 & 0.7809 & 0.7413 & $2.72 \mathrm{E}-05$ \\
\hline & error $(\%)$ & -2.79 & -9.18 & 2.97 & -2.24 & 7.69 \\
\hline & retrieved parameters & $\sqrt{\left\langle x_{\mathrm{CO}}^{\prime 2}\right\rangle}$ & $\left\langle T^{\prime} x_{\mathrm{CO}}^{\prime}\right\rangle(\mathrm{K})$ & $\left\langle x_{\mathrm{CO}_{2}}^{\prime} x_{\mathrm{CO}}^{\prime}\right\rangle$ & $\left\langle x_{\mathrm{H}_{2} \mathrm{O}}^{\prime} x_{\mathrm{CO}}^{\prime}\right\rangle$ & $\Lambda / L$ \\
\hline & actual values & 0.0201 & 3.0335 & - & $1.01 \mathrm{E}-04$ & 0.100 \\
\hline \multirow{2}{*}{ II } & retrieved & - & - & - & - & 0.102 \\
\hline & error $(\%)$ & - & - & - & - & 2.14 \\
\hline \multirow{2}{*}{ III } & retrieved & 0.0157 & 3.0176 & - & $1.24 \mathrm{E}-04$ & - \\
\hline & error $(\%)$ & -22.04 & -0.52 & - & 22.23 & - \\
\hline
\end{tabular}

$\left\langle x_{\mathrm{CO}_{2}}^{\prime 2}\right\rangle,\left\langle x_{\mathrm{H}_{2} \mathrm{O}}^{\prime 2}\right\rangle,\left\langle T^{\prime} x_{\mathrm{CO}_{2}}^{\prime}\right\rangle,\left\langle T^{\prime} x_{\mathrm{H}_{2} \mathrm{O}}^{\prime}\right\rangle,\left\langle x_{\mathrm{CO}_{2}}^{\prime} x_{\mathrm{H}_{2} \mathrm{O}}^{\prime}\right\rangle$ and $\Lambda$ are retrieved from spectral interval II; and $\left\langle x_{\mathrm{CO}}^{\prime 2}\right\rangle,\left\langle T^{\prime} x_{\mathrm{CO}}^{\prime}\right\rangle$, and $\left\langle x_{\mathrm{H}_{2} \mathrm{O}}^{\prime} x_{\mathrm{CO}}^{\prime}\right\rangle$ are retrieved from spectral interval III.

Tables 4 and 5 show inverse calculation results for mean temperatures of $1000 \mathrm{~K}$ and $2000 \mathrm{~K}$, respectively. Except parameters for $\mathrm{CO}$, all other parameters are retrieved fairly accurately. Although the relative errors for retrieving $\mathrm{CO}$ parameters are large, considering the facts that the $\mathrm{CO}$ concentration is small and thus the $\mathrm{CO}$ band in spectral interval III is weak, the results are quite good. Tables 6 and 7 show inverse calculation results for turbulent gas fields with $5 \%$ and $15 \%$ turbulent fluctuations, respectively. Because the calculation of turbulent transmission is based on the local linearization of the absorption coefficient with temperature and 

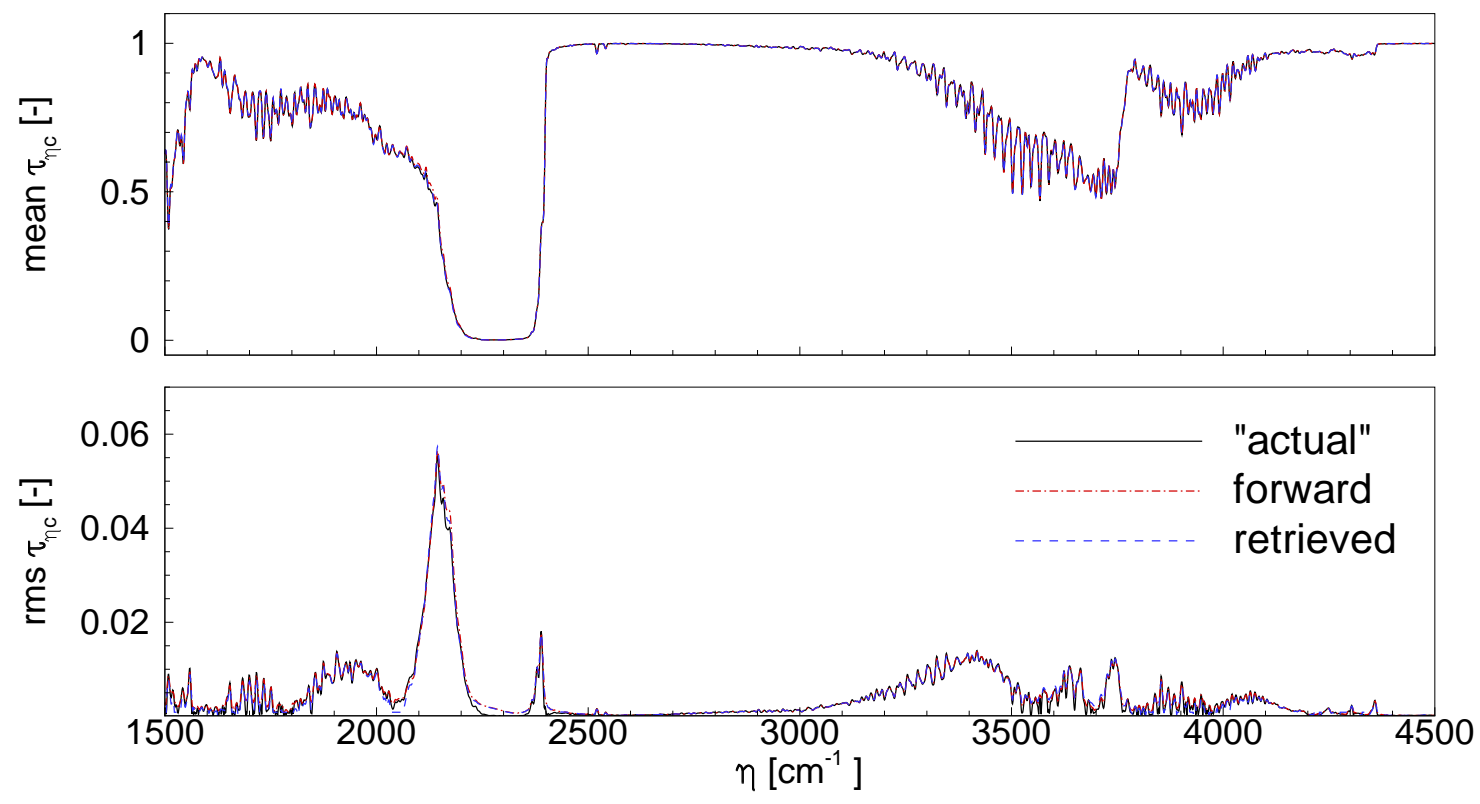

Figure 5: Comparison of retrieved transmissivity and its rms spectra with the "actual" spectra and forward spectra calculated by the actual turbulent scalars for a gas mixture of $5 \% \mathrm{CO}_{2}+5 \% \mathrm{H}_{2} \mathrm{O}+20 \% \mathrm{CO}$

gas concentrations, the model is more accurate for smaller turbulent fluctuations. Consequently, the inverse results are more accurate when the turbulent fluctuation is as small as 5\%, which is shown in Table 6 . When the turbulent fluctuations are large, the linear assumption of the absorption coefficient with temperature and gas concentrations introduces more error in the forward calculation and, therefore, the inverse results are less accurate. As shown in Table 7 the retrieved mean concentrations for $\mathrm{CO}_{2}$ and $\mathrm{CO}$ become less accurate when the turbulent fluctuation is as large as $15 \%$. Table 8 shows the inverse calculation results for turbulent fields with a integral length scale of $\Lambda=0.2 \mathrm{~m}(\Lambda / L=0.2)$. The integral length scale $\Lambda$ provides a constant scaling over the variance of transmissivity, as shown in Eq. 21), which is easer to be retrieved for any $\Lambda$. The results in Table 8 show that the proposed methods also work for a different $\Lambda$.

\subsubsection{Pressure path length}

For a homogeneous gas column, the transmissivity of the gas is defined as

$$
\tau_{\eta}=e^{-\kappa_{P \eta} P x L}
$$

where $\kappa_{P \eta}$ is the pressure-based absorption coefficient, $P$ is the total pressure, $x$ is the gas concentration and $L$ is the length of the gas column. Because total pressure, species concentration and gas path length have similar effects on transmissivity, we define the pressure path length PxL as the product of partial pressure and gas path length. Although, for a gas mixture, the $P x L$ effects on transmissivity are different for different species, the trends are similar. To simplify the analysis for a gas mixture, the same definition is adopted here while $x$ becomes the summation of the concentrations for all absorbing species.

As indicated in Eq. (24), increasing the pressure path length $P x L$ decreases transmissivity and eventually, the transmissivity tends toward zero and becomes saturated, which makes the spectrum less spectrally distinct and the inverse calculations less accurate. In spectral interval I, all three gases have absorption bands, but they do not totally overlap with each other. Using this part of the spectrum one may still retrieve the mean temperature and species concentrations if the spectrum is not totally saturated. However, in spectral interval II, $\mathrm{H}_{2} \mathrm{O}$ absorption overlaps the entire $\mathrm{CO}_{2}$ band. As total pressure $P$ or gas path length $L$ increases, the transmissivity spectrum becomes saturated and masks all information for $\mathrm{CO}_{2}$. Thus, for large mixture pressure path length, the turbulent 
Table 4: Inverse calculation results for conditions: $5 \% \mathrm{CO}_{2}+20 \% \mathrm{H}_{2} \mathrm{O}+5 \% \mathrm{CO}, 1000 \mathrm{~K}, P=1$ bar, $L=100 \mathrm{~cm}$, $\sqrt{\left\langle z^{\prime 2}\right\rangle} /\langle z\rangle=10 \%$ and $\Lambda / L=0.1$

\begin{tabular}{|c|c|c|c|c|c|c|}
\hline & retrieved parameters & $\langle T\rangle(\mathrm{K})$ & $\left\langle x_{\mathrm{CO}_{2}}\right\rangle$ & $\left\langle x_{\mathrm{H}_{2} \mathrm{O}}\right\rangle$ & $\left\langle x_{\mathrm{CO}}\right\rangle$ & $\sqrt{\left\langle T^{\prime 2}\right\rangle}(\mathrm{K})$ \\
\hline \multirow{3}{*}{ I } & actual values & 999 & 0.0499 & 0.1998 & 0.0499 & 101.1 \\
\hline & retrieved & 1018 & 0.0505 & 0.1988 & 0.0526 & - \\
\hline & error(\%) & 1.89 & 1.19 & -0.52 & 5.36 & - \\
\hline \multirow{3}{*}{ II } & retrieved & 995 & 0.0495 & 0.2013 & - & 101.8 \\
\hline & error(\%) & -0.35 & -0.91 & 0.75 & - & 0.67 \\
\hline & retrieved parameters & $\sqrt{\left\langle x_{\mathrm{CO}_{2}}^{\prime 2}\right\rangle}$ & $\sqrt{\left\langle x_{\mathrm{H}_{2} \mathrm{O}}^{\prime 2}\right\rangle}$ & $\left\langle T^{\prime} x_{\mathrm{CO}_{2}}^{\prime}\right\rangle(\mathrm{K})$ & $\left\langle T^{\prime} x_{\mathrm{H}_{2} \mathrm{O}}^{\prime}\right\rangle(\mathrm{K})$ & $\left\langle x_{\mathrm{CO}_{2}}^{\prime} x_{\mathrm{H}_{2} \mathrm{O}}^{\prime}\right\rangle$ \\
\hline \multirow{5}{*}{ II } & actual values & 0.0051 & 0.0202 & 0.5115 & 2.0459 & $1.02 \mathrm{E}-04$ \\
\hline & retrieved & 0.0047 & 0.0191 & 0.5183 & 2.0602 & $1.07 \mathrm{E}-04$ \\
\hline & error $(\%)$ & -6.96 & -5.82 & 1.34 & 0.70 & 4.44 \\
\hline & retrieved parameters & $\sqrt{\left\langle x_{\mathrm{CO}}^{\prime 2}\right\rangle}$ & $\left\langle T^{\prime} x_{\mathrm{CO}}^{\prime}\right\rangle(\mathrm{K})$ & $\left\langle x_{\mathrm{CO}_{2}}^{\prime} x_{\mathrm{CO}}^{\prime}\right\rangle$ & $\left\langle x_{\mathrm{H}_{2} \mathrm{O}}^{\prime} x_{\mathrm{CO}}^{\prime}\right\rangle$ & $\Lambda / L$ \\
\hline & actual values & 0.0051 & 0.5115 & - & $1.02 \mathrm{E}-04$ & 0.100 \\
\hline \multirow{2}{*}{ II } & retrieved & - & - & - & - & 0.104 \\
\hline & error $(\%)$ & - & - & - & - & 3.90 \\
\hline \multirow{2}{*}{ III } & retrieved & 0.0072 & 0.6061 & - & $1.46 \mathrm{E}-04$ & - \\
\hline & error $(\%)$ & 42.86 & 18.50 & - & 42.51 & - \\
\hline
\end{tabular}

Table 5: Inverse calculation results for conditions: $5 \% \mathrm{CO}_{2}+20 \% \mathrm{H}_{2} \mathrm{O}+5 \% \mathrm{CO}, 2000 \mathrm{~K}, P=1$ bar, $L=100 \mathrm{~cm}$, $\sqrt{\left\langle z^{\prime 2}\right\rangle} /\langle z\rangle=10 \%$ and $\Lambda / L=0.1$

\begin{tabular}{|c|c|c|c|c|c|c|}
\hline & retrieved parameters & $\langle T\rangle(\mathrm{K})$ & $\left\langle x_{\mathrm{CO}_{2}}\right\rangle$ & $\left\langle x_{\mathrm{H}_{2} \mathrm{O}}\right\rangle$ & $\left\langle x_{\mathrm{CO}}\right\rangle$ & $\sqrt{\left\langle T^{\prime 2}\right\rangle}(\mathrm{K})$ \\
\hline \multirow{3}{*}{ I } & actual values & 1998 & 0.0499 & 0.1998 & 0.0499 & 201.5 \\
\hline & retrieved & 2003 & 0.0510 & 0.1987 & 0.0514 & - \\
\hline & error(\%) & 0.26 & 2.14 & -0.53 & 2.93 & - \\
\hline \multirow{3}{*}{ II } & retrieved & 1976 & 0.0489 & 0.1981 & - & 203.6 \\
\hline & error $(\%)$ & -1.11 & -2.06 & -0.82 & - & 1.01 \\
\hline & retrieved parameters & $\sqrt{\left\langle x_{\mathrm{CO}_{2}}^{\prime 2}\right\rangle}$ & $\sqrt{\left\langle x_{\mathrm{H}_{2} \mathrm{O}}^{\prime 2}\right\rangle}$ & $\left\langle T^{\prime} x_{\mathrm{CO}_{2}}^{\prime}\right\rangle(\mathrm{K})$ & $\left\langle T^{\prime} x_{\mathrm{H}_{2} \mathrm{O}}^{\prime}\right\rangle(\mathrm{K})$ & $\left\langle x_{\mathrm{CO}_{2}}^{\prime} x_{\mathrm{H}_{2} \mathrm{O}}^{\prime}\right\rangle$ \\
\hline \multirow{5}{*}{ II } & actual values & 0.0050 & 0.0202 & 1.0152 & 4.0607 & $1.02 \mathrm{E}-04$ \\
\hline & retrieved & 0.0049 & 0.0185 & 1.1153 & 3.9138 & $1.13 \mathrm{E}-04$ \\
\hline & error(\%) & -3.38 & -8.31 & 9.86 & -3.62 & 10.88 \\
\hline & retrieved parameters & $\sqrt{\left\langle x_{\mathrm{CO}}^{\prime 2}\right\rangle}$ & $\left\langle T^{\prime} x_{\mathrm{CO}}^{\prime}\right\rangle(\mathrm{K})$ & $\left\langle x_{\mathrm{CO}_{2}}^{\prime} x_{\mathrm{CO}}^{\prime}\right\rangle$ & $\left\langle x_{\mathrm{H}_{2} \mathrm{O}}^{\prime} x_{\mathrm{CO}}^{\prime}\right\rangle$ & $\Lambda / L$ \\
\hline & actual values & 0.0050 & 1.0152 & - & $1.02 \mathrm{E}-04$ & 0.100 \\
\hline \multirow{2}{*}{ II } & retrieved & - & - & - & - & 0.104 \\
\hline & error(\%) & - & - & - & - & 4.12 \\
\hline \multirow{2}{*}{ III } & retrieved & 0.0029 & 1.1602 & - & $1.12 \mathrm{E}-04$ & - \\
\hline & error $(\%)$ & -41.79 & 14.29 & - & 10.41 & - \\
\hline
\end{tabular}

scalars related to $\mathrm{CO}_{2}$ and $\mathrm{H}_{2} \mathrm{O}$ cannot be retrieved using spectral interval II. Figure 6 (a) shows the spectral absorption coefficient for a gas mixture of $5 \% \mathrm{CO}_{2}, 20 \% \mathrm{H}_{2} \mathrm{O}$ and $5 \% \mathrm{CO}$ at $10 \mathrm{bar}$ and $1500 \mathrm{~K}$, and the "actual" transmissivity and its rms spectra are shown in Fig. 6(b). The mixture pressure path length is $300 \mathrm{bar} \cdot \mathrm{cm}$. As shown in the figures, the spectrum is saturated over most parts of spectral intervals I and II, neither mean or rms values can be retrieved.

For all previous tested cases, the mixture $P x L$ was $30 \mathrm{bar} \cdot \mathrm{cm}$. Our numerical tests indicate that as long as the mixture $P x L$ is $60 \mathrm{bar} \cdot \mathrm{cm}$ or less, the proposed method is valid. Table 9 shows the inverse calculation results for a total pressure of 10 bar and a gas mixture of $10 \% \mathrm{CO}_{2}+10 \% \mathrm{H}_{2} \mathrm{O}+10 \% \mathrm{CO}$ with $\mathrm{N}_{2}$ in a $20 \mathrm{~cm}$ long gas column (the mixture $P x L=60 \mathrm{bar} \cdot \mathrm{cm}$ ), a mean temperature of $1500 \mathrm{~K}$, turbulent fluctuations of $10 \%$ and a turbulence integral length scale of $\Lambda / L=0.1$. All turbulent scalars were well retrieved. As stated before, the synthetic "measurements" were not perturbed by random noise. Therefore, as long as the spectra are not 
Table 6: Inverse calculation results for conditions: $5 \% \mathrm{CO}_{2}+20 \% \mathrm{H}_{2} \mathrm{O}+5 \% \mathrm{CO}, 1500 \mathrm{~K}, P=1$ bar, $L=100 \mathrm{~cm}$, $\sqrt{\left\langle z^{\prime 2}\right\rangle} /\langle z\rangle=5 \%$ and $\Lambda / L=0.1$

\begin{tabular}{|c|c|c|c|c|c|c|}
\hline & retrieved parameters & $\langle T\rangle(\mathrm{K})$ & $\left\langle x_{\mathrm{CO}_{2}}\right\rangle$ & $\left\langle x_{\mathrm{H}_{2} \mathrm{O}}\right\rangle$ & $\left\langle x_{\mathrm{CO}}\right\rangle$ & $\sqrt{\left\langle T^{\prime 2}\right\rangle}(\mathrm{K})$ \\
\hline \multirow{3}{*}{ I } & actual values & 1499 & 0.1999 & 0.0500 & 0.0500 & 75.9 \\
\hline & retrieved & 1507 & 0.2024 & 0.0500 & 0.0502 & - \\
\hline & error(\%) & 0.50 & 1.27 & 0.06 & 0.54 & - \\
\hline \multirow{3}{*}{ II } & retrieved & 1504 & 0.1999 & 0.0500 & - & 76.0 \\
\hline & error(\%) & 0.32 & -0.02 & 0.12 & - & 0.22 \\
\hline & retrieved parameters & $\sqrt{\left\langle x_{\mathrm{CO}_{2}}^{\prime 2}\right\rangle}$ & $\sqrt{\left\langle x_{\mathrm{H}_{2} \mathrm{O}}^{\prime 2}\right\rangle}$ & $\left\langle T^{\prime} x_{\mathrm{CO}_{2}}^{\prime}\right\rangle(\mathrm{K})$ & $\left\langle T^{\prime} x_{\mathrm{H}_{2} \mathrm{O}}^{\prime}\right\rangle(\mathrm{K})$ & $\left\langle x_{\mathrm{CO}_{2}}^{\prime} x_{\mathrm{H}_{2} \mathrm{O}}^{\prime}\right\rangle$ \\
\hline \multirow{5}{*}{ II } & actual values & 0.0101 & 0.0025 & 0.7672 & 0.1918 & $2.56 \mathrm{E}-05$ \\
\hline & retrieved & 0.0103 & 0.0024 & 0.7833 & 0.1916 & $2.28 \mathrm{E}-05$ \\
\hline & error $(\%)$ & 2.30 & -6.45 & 2.09 & -0.13 & -10.72 \\
\hline & retrieved parameters & $\sqrt{\left\langle x_{\mathrm{CO}}^{\prime 2}\right\rangle}$ & $\left\langle T^{\prime} x_{\mathrm{CO}}^{\prime}\right\rangle(\mathrm{K})$ & $\left\langle x_{\mathrm{CO}_{2}}^{\prime} x_{\mathrm{CO}}^{\prime}\right\rangle$ & $\left\langle x_{\mathrm{H}_{2} \mathrm{O}}^{\prime} x_{\mathrm{CO}}^{\prime}\right\rangle$ & $\Lambda / L$ \\
\hline & actual values & 0.0025 & 0.1918 & - & $6.39 \mathrm{E}-06$ & 0.100 \\
\hline \multirow{2}{*}{ II } & retrieved & - & - & - & - & 0.103 \\
\hline & error $(\%)$ & - & - & - & - & 3.19 \\
\hline \multirow{2}{*}{ III } & retrieved & 0.0027 & 0.1995 & - & $6.14 \mathrm{E}-06$ & - \\
\hline & error $(\%)$ & 4.94 & 4.03 & - & -3.96 & - \\
\hline
\end{tabular}

Table 7: Inverse calculation results for conditions: $5 \% \mathrm{CO}_{2}+20 \% \mathrm{H}_{2} \mathrm{O}+5 \% \mathrm{CO}, 1500 \mathrm{~K}, P=1$ bar, $L=100 \mathrm{~cm}$, $\sqrt{\left\langle z^{\prime 2}\right\rangle} /\langle z\rangle=15 \%$ and $\Lambda / L=0.1$

\begin{tabular}{|c|c|c|c|c|c|c|}
\hline & retrieved parameters & $\langle T\rangle(\mathrm{K})$ & $\left\langle x_{\mathrm{CO}_{2}}\right\rangle$ & $\left\langle x_{\mathrm{H}_{2} \mathrm{O}}\right\rangle$ & $\left\langle x_{\mathrm{CO}}\right\rangle$ & $\sqrt{\left\langle T^{\prime 2}\right\rangle}(\mathrm{K})$ \\
\hline \multirow{3}{*}{ I } & actual values & 1501 & 0.0500 & 0.2001 & 0.0500 & 223.8 \\
\hline & retrieved & 1539 & 0.0525 & 0.1986 & 0.0554 & - \\
\hline & error(\%) & 2.55 & 5.02 & -0.73 & 10.76 & - \\
\hline \multirow{3}{*}{ II } & retrieved & 1492 & 0.0490 & 0.2014 & - & 218.0 \\
\hline & $\operatorname{error}(\%)$ & -0.60 & -2.05 & 0.64 & - & -2.58 \\
\hline & retrieved parameters & $\sqrt{\left\langle x_{\mathrm{CO}_{2}}^{\prime 2}\right\rangle}$ & $\sqrt{\left\langle x_{\mathrm{H}_{2} \mathrm{O}}^{\prime 2}\right\rangle}$ & $\left\langle T^{\prime} x_{\mathrm{CO}_{2}}^{\prime}\right\rangle(\mathrm{K})$ & $\left\langle T^{\prime} x_{\mathrm{H}_{2} \mathrm{O}}^{\prime}\right\rangle(\mathrm{K})$ & $\left\langle x_{\mathrm{CO}_{2}}^{\prime} x_{\mathrm{H}_{2} \mathrm{O}}^{\prime}\right\rangle$ \\
\hline \multirow{5}{*}{ II } & actual values & 0.0075 & 0.0298 & 1.6699 & 6.6794 & $2.23 \mathrm{E}-04$ \\
\hline & retrieved & 0.0034 & 0.0276 & 1.5349 & 6.4999 & $2.81 \mathrm{E}-04$ \\
\hline & error $(\%)$ & -54.57 & -7.51 & -8.08 & -2.69 & 26.28 \\
\hline & retrieved parameters & $\sqrt{\left\langle x_{\mathrm{CO}}^{\prime 2}\right\rangle}$ & $\left\langle T^{\prime} x_{\mathrm{CO}}^{\prime}\right\rangle(\mathrm{K})$ & $\left\langle x_{\mathrm{CO}_{2}}^{\prime} x_{\mathrm{CO}}^{\prime}\right\rangle$ & $\left\langle x_{\mathrm{H}_{2} \mathrm{O}}^{\prime} x_{\mathrm{CO}}^{\prime}\right\rangle$ & $\Lambda / L$ \\
\hline & actual values & 0.0075 & 1.6699 & - & $2.23 \mathrm{E}-04$ & 0.100 \\
\hline \multirow{2}{*}{ II } & retrieved & - & - & - & - & 0.093 \\
\hline & error $(\%)$ & - & - & - & - & -6.72 \\
\hline \multirow{2}{*}{ III } & retrieved & 0.0064 & 1.5376 & - & $2.00 \mathrm{E}-04$ & - \\
\hline & error $(\%)$ & -13.73 & -7.92 & - & -10.02 & - \\
\hline
\end{tabular}

saturated, turbulent scalars can be retrieved. Thus there is no identified minimum mixture $P x L$ for the present study.

\section{Conclusions}

Inverse radiation models were developed for temperature and species concentrations determination in turbulent combustion gas mixtures. Synthetic transmissivity spectra were created for turbulent gas mixtures containing $\mathrm{CO}_{2}, \mathrm{H}_{2} \mathrm{O}$ and $\mathrm{CO}$, which were used to validate the inverse radiation models. For turbulent transmission measurements of a gas mixture, time-averaged temperature and species concentrations can be retrieved from the $1800-2500 \mathrm{~cm}^{-1}$ spectral interval; all parameters related to $\mathrm{CO}_{2}$ and $\mathrm{H}_{2} \mathrm{O}$ plus turbulent length scale can be retrieved from the $3200-3800 \mathrm{~cm}^{-1}$ spectral interval; and the remaining parameters related to $\mathrm{CO}$ can be 
Table 8: Inverse calculation results for conditions: $5 \% \mathrm{CO}_{2}+20 \% \mathrm{H}_{2} \mathrm{O}+5 \% \mathrm{CO}, 1500 \mathrm{~K}, P=1$ bar, $L=100 \mathrm{~cm}$, $\sqrt{\left\langle z^{\prime 2}\right\rangle} /\langle z\rangle=10 \%$ and $\Lambda / L=0.2$

\begin{tabular}{|c|c|c|c|c|c|c|}
\hline & retrieved parameters & $\langle T\rangle(\mathrm{K})$ & $\left\langle x_{\mathrm{CO}_{2}}\right\rangle$ & $\left\langle x_{\mathrm{H}_{2} \mathrm{O}}\right\rangle$ & $\left\langle x_{\mathrm{CO}}\right\rangle$ & $\sqrt{\left\langle T^{\prime 2}\right\rangle}(\mathrm{K})$ \\
\hline \multirow{3}{*}{ I } & actual values & 1501 & 0.0500 & 0.2002 & 0.0500 & 147.9 \\
\hline & retrieved & 1519 & 0.0512 & 0.1994 & 0.0526 & - \\
\hline & error(\%) & 1.17 & 2.27 & -0.39 & 5.14 & - \\
\hline \multirow{4}{*}{ II } & retrieved & 1494 & 0.0494 & 0.2006 & - & 143.3 \\
\hline & error $(\%)$ & -0.49 & -1.19 & 0.20 & - & -3.08 \\
\hline & retrieved parameters & $\sqrt{\left\langle x_{\mathrm{CO}_{2}}^{\prime 2}\right\rangle}$ & $\sqrt{\left\langle x_{\mathrm{H}_{2} \mathrm{O}}^{\prime 2}\right\rangle}$ & $\left\langle T^{\prime} x_{\mathrm{CO}_{2}}^{\prime}\right\rangle(\mathrm{K})$ & $\left\langle T^{\prime} x_{\mathrm{H}_{2} \mathrm{O}}^{\prime}\right\rangle(\mathrm{K})$ & $\left\langle x_{\mathrm{CO}_{2}}^{\prime} x_{\mathrm{H}_{2} \mathrm{O}}^{\prime}\right\rangle$ \\
\hline & actual values & 0.0049 & 0.0197 & 0.7289 & 2.9158 & $9.72 \mathrm{E}-05$ \\
\hline \multirow{4}{*}{ II } & retrieved & 0.0038 & 0.0173 & 0.7224 & 2.6767 & $1.12 \mathrm{E}-04$ \\
\hline & error $(\%)$ & -23.33 & -12.08 & -0.89 & -8.20 & 15.09 \\
\hline & retrieved parameters & $\sqrt{\left\langle x_{\mathrm{CO}}^{\prime 2}\right\rangle}$ & $\left\langle T^{\prime} x_{\mathrm{CO}}^{\prime}\right\rangle(\mathrm{K})$ & $\left\langle x_{\mathrm{CO}_{2}}^{\prime} x_{\mathrm{CO}}^{\prime}\right\rangle$ & $\left\langle x_{\mathrm{H}_{2} \mathrm{O}}^{\prime} x_{\mathrm{CO}}^{\prime}\right\rangle$ & $\Lambda / L$ \\
\hline & actual values & 0.0049 & 0.7289 & - & $9.72 \mathrm{E}-05$ & 0.200 \\
\hline \multirow{2}{*}{ II } & retrieved & - & - & - & - & 0.206 \\
\hline & error $(\%)$ & - & - & - & - & 3.24 \\
\hline \multirow{2}{*}{ III } & retrieved & 0.0059 & 0.7458 & - & $8.05 \mathrm{E}-05$ & - \\
\hline & error $(\%)$ & 19.50 & 2.31 & - & -17.16 & - \\
\hline
\end{tabular}
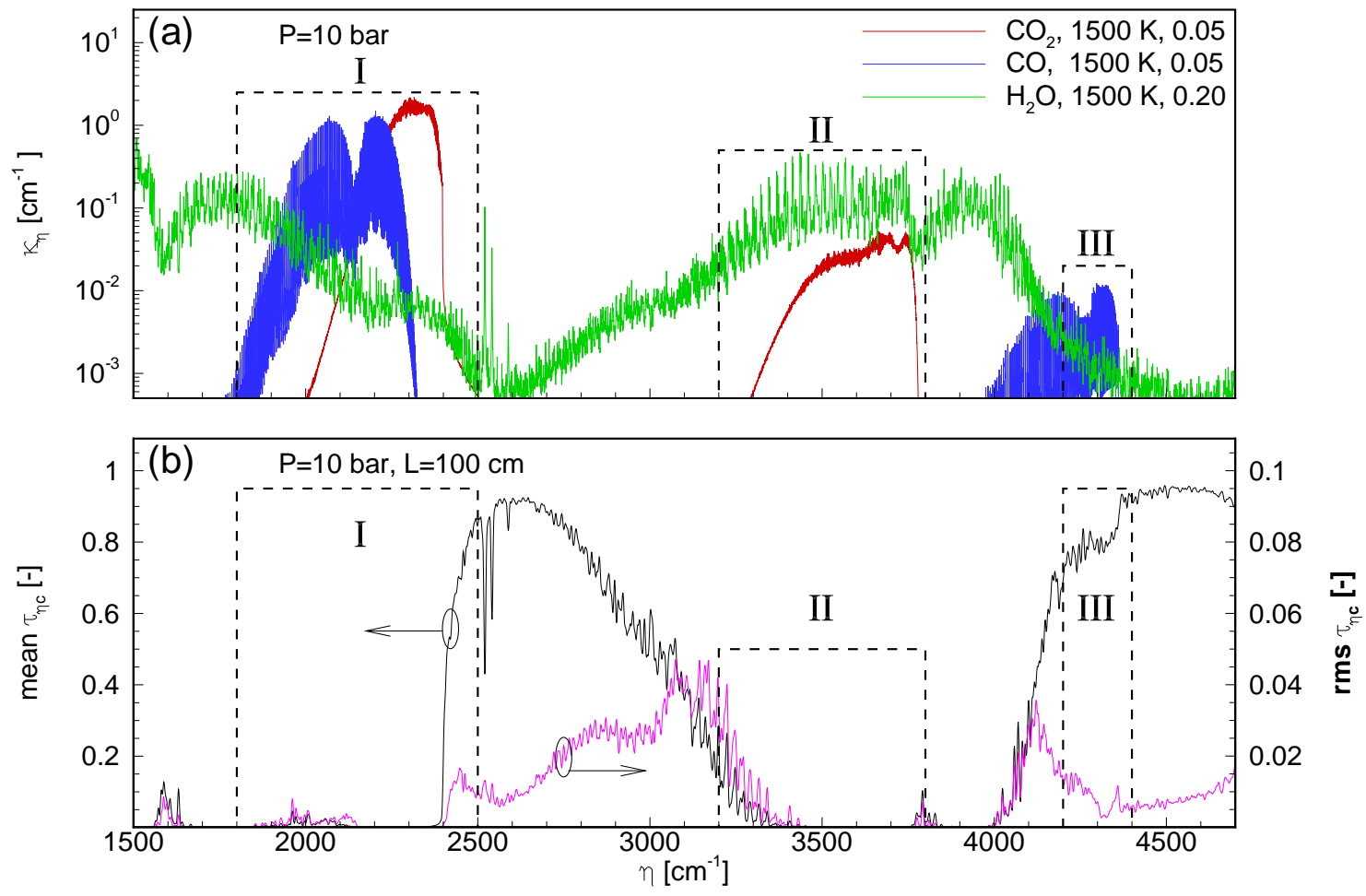

Figure 6: (a) spectral absorption coefficient and (b) "actual" transmissivity and its rms spectra for conditions: $5 \% \mathrm{CO}_{2}+20 \% \mathrm{H}_{2} \mathrm{O}+5 \% \mathrm{CO}, 1500 \mathrm{~K}, P=10$ bar, $L=100 \mathrm{~cm}, \sqrt{\left\langle z^{2}\right\rangle} /\langle z\rangle=10 \%$ and $\Lambda / L=0.1$

retrieved from the $4200-4400 \mathrm{~cm}^{-1}$ spectral interval. A series of numerical tests were conducted for different temperatures, combination of species concentrations, turbulent intensities, turbulent length scales and mixture 
Table 9: Inverse calculation results for conditions: $10 \% \mathrm{CO}_{2}+10 \% \mathrm{H}_{2} \mathrm{O}+10 \% \mathrm{CO}, 1500 \mathrm{~K}, P=10$ bar, $L=20$ $\mathrm{cm}, \sqrt{\left\langle z^{\prime 2}\right\rangle} /\langle z\rangle=10 \%$ and $\Lambda / L=0.1$

\begin{tabular}{|c|c|c|c|c|c|c|}
\hline & retrieved parameters & $\langle T\rangle(\mathrm{K})$ & $\left\langle x_{\mathrm{CO}_{2}}\right\rangle$ & $\left\langle x_{\mathrm{H}_{2} \mathrm{O}}\right\rangle$ & $\left\langle x_{\mathrm{CO}}\right\rangle$ & $\sqrt{\left\langle T^{\prime 2}\right\rangle}(\mathrm{K})$ \\
\hline \multirow{3}{*}{ I } & actual values & 1495 & 0.0997 & 0.0997 & 0.0997 & 150.8 \\
\hline & retrieved & 1511 & 0.1067 & 0.0998 & 0.1006 & - \\
\hline & error(\%) & 1.09 & 7.01 & 0.10 & 0.93 & - \\
\hline \multirow{3}{*}{ II } & retrieved & 1494 & 0.0986 & 0.1002 & - & 152.2 \\
\hline & error $(\%)$ & -0.08 & -1.05 & 0.52 & - & 0.90 \\
\hline & retrieved parameters & $\sqrt{\left\langle x_{\mathrm{CO}_{2}}^{\prime 2}\right\rangle}$ & $\sqrt{\left\langle x_{\mathrm{H}_{2} \mathrm{O}}^{\prime 2}\right\rangle}$ & $\left\langle T^{\prime} x_{\mathrm{CO}_{2}}^{\prime}\right\rangle(\mathrm{K})$ & $\left\langle T^{\prime} x_{\mathrm{H}_{2} \mathrm{O}}^{\prime}\right\rangle(\mathrm{K})$ & $\left\langle x_{\mathrm{CO}_{2}}^{\prime} x_{\mathrm{H}_{2} \mathrm{O}}^{\prime}\right\rangle$ \\
\hline \multirow{5}{*}{ II } & actual values & 0.0101 & 0.0101 & 1.5167 & 1.5167 & $1.01 \mathrm{E}-04$ \\
\hline & retrieved & 0.0107 & 0.0092 & 1.6179 & 1.4779 & $1.01 \mathrm{E}-04$ \\
\hline & $\operatorname{error}(\%)$ & 6.20 & -8.45 & 6.67 & -2.56 & 0.29 \\
\hline & retrieved parameters & $\sqrt{\left\langle x_{\mathrm{CO}}^{\prime 2}\right\rangle}$ & $\left\langle T^{\prime} x_{\mathrm{CO}}^{\prime}\right\rangle(\mathrm{K})$ & $\left\langle x_{\mathrm{CO}_{2}}^{\prime} x_{\mathrm{CO}}^{\prime}\right\rangle$ & $\left\langle x_{\mathrm{H}_{2} \mathrm{O}}^{\prime} x_{\mathrm{CO}}^{\prime}\right\rangle$ & $\Lambda / L$ \\
\hline & actual values & 0.0101 & 1.5167 & - & $1.01 \mathrm{E}-04$ & 0.100 \\
\hline \multirow{2}{*}{ II } & retrieved & - & - & - & - & 0.103 \\
\hline & $\operatorname{error}(\%)$ & - & - & - & - & 3.36 \\
\hline \multirow{2}{*}{ III } & retrieved & 0.0115 & 1.6013 & - & $1.18 \mathrm{E}-04$ & - \\
\hline & error $(\%)$ & 14.66 & 5.57 & - & 16.53 & - \\
\hline
\end{tabular}

pressure path lengths. Results show that temperature and species concentrations and other turbulent scalars can be retrieved well from turbulent transmissivity measurements as long as the turbulent intensity is below $15 \%$ and the pressure path length for the gas mixture is less than $60 \mathrm{bar} \cdot \mathrm{cm}$.

\section{Acknowledgment}

The authors gratefully acknowledge the support from National Science Foundation Grant No. CBET-0966627.

\section{References}

[1] R. J. Anderson, P. R. Griffiths, Determination of rotational temperatures of diatomic molecules from absorption spectra measured at moderate resolution, J Quant Spectrosc Radiat Transf 17 (1977) 393-401.

[2] L. A. Gross, P. R. Griffiths, Temperature estimation of carbon dioxide by infrared absorption spectrometry at medium resolution, J Quant Spectrosc Radiat Transf 39 (2) (1988) 131-138.

[3] P. R. Solomon, P. E. Best, R. M. Carangelo, J. R. Markham, P.-L. Chien, R. J. Santoro, H. G. Semerjian, Ft-ir emission/transmission spectroscopy for in situ combustion diagnostics, Proc Comb Inst 21 (1987) 1763-1771.

[4] P. E. Best, P. L. Chien, R. M. Carangelo, P. R. Solomon, M. Danchak, I. Ilovici, Tomographic reconstruction of $\mathrm{ft}$-ir emission and transmission spectra in a sooting laminar diffusion flame: Species concentrations and temperatures, Combust Flame 85 (1991) 309-314.

[5] S.-W. Woo, T.-H. Song, Measurement of gas temperature profile using spectral intensity from $\mathrm{co}_{2} 4.3 \mu \mathrm{m}$ band, International journal of thermal sciences 41 (9) (2002) 883-890.

[6] H. K. Kim, T.-H. Song, Characteristics of srs inversion for measurement of temperature and $\mathrm{co}_{2}$ concentration profile of a combustion gas layer, J Quant Spectrosc Radiat Transf 86 (2) (2004) 181-199.

[7] H. K. Kim, T.-H. Song, Determination of the gas temperature profile in a large-scale furnace using a fast/efficient inversion scheme for the SRS technique, J Quant Spectrosc Radiat Transf 93 (2005) 369381. 
[8] T.-H. Song, Spectral remote sensing for furnaces and flames, Heat Transfer Engineering 29 (4) (2008) 417-428.

[9] A. A. Townsend, The effects of radiative transfer on turbulent flow of a stratified fluid, J Fluid Mech 4 (1958) 361-375.

[10] T.-H. Song, R. Viskanta, Interaction of radiation with turbulence: Application to a combustion system, J Thermoph Heat Transfer 1 (1987) 56-62.

[11] A. Soufiani, P. Mignon, J. Taine, Radiation-turbulence interaction in channel flows of infrared active gases, in: Proceedings of the International Heat Transfer Conference, Vol. 6, ASME, 1990, pp. 403-408.

[12] R. J. Hall, A. Vranos, Efficient calculations of gas radiation from turbulent flames, Intl J Heat Mass Transfer 37 (17) (1994) 2745-2750.

[13] S. Tieszen, On the fluid mechanics of fires, Ann. Rev. Fluid Mech. 33 (2001) 67-92.

[14] P. J. Hommert, R. Viskanta, A. M. Mellor, Flame temperature measurements by spectral remote sensing, Combust Flame 30 (1977) 295-308.

[15] D. R. Buchele, Computer program for calculation of a gas temperature profile by infrared emission: Absorption spectroscopy, NASA-TM-73848.

[16] T. Ren, M. F. Modest, Temperature profile inversion from carbon-dioxide spectral intensities through Tikhonov regularization, J Thermoph Heat Transfer doi: 10.2514/1.T4561.

[17] T. Ren, M. F. Modest, A. Fateev, S. Clausen, An inverse radiation model for optical determination of temperature and species concentration: development and validation, J Quant Spectrosc Radiat Transf 151 (0) (2015) 198-209.

[18] J. Lim, Y. Sivathanu, J. Ji, J. Gore, Estimating scalars from spectral radiation measurements in a homogeneous hot gas layer, Combust Flame 137 (2004) 222-229.

[19] W. L. Grosshandler, Radiative transfer in nonhomogeneous gases: A simplified approach, Intl J Heat Mass Transfer 23 (1980) 1447-1457.

[20] J. Ji, Experimental and theoretical study of the spectral radiation characteristics of lean premixed flames, Ph.D. thesis, Purdue University, West Lafayette, IN (2000).

[21] J. Ji, J. P. Gore, Y. R. Sivathanu, J. Lim, Fast infrared array spectrometer with a thermoelectrically cooled 160-element pbse detector, Review of scientific instruments 75 (2) (2004) 333-339.

[22] S. M. Jeng, M. C. Lai, G. M. Faeth, Nonluminous radiation in turbulent buoyant axisymmetric flames, Combust Sci Technol 40 (1984) 41-53.

[23] J. P. Gore, G. M. Faeth, Structure and spectral radiation properties of turbulent ethylene/air diffusion flames, in: Proceedings of the Twenty-First Symposium (International) on Combustion, 1986, pp. 15211531.

[24] J. P. Gore, S. M. Jeng, G. M. Faeth, Spectral and total radiation properties of turbulent carbon monoxide/air diffusion flames, AIAA J 25 (2) (1987) 339-345.

[25] J. P. Gore, S. M. Jeng, G. M. Faeth, Spectral and total radiation properties of turbulent hydrogen/air diffusion flames, ASME J Heat Transfer 109 (1987) 165-171.

[26] M. E. Kounalakis, J. P. Gore, G. M. Faeth, Turbulence/radiation interactions in nonpremixed hydrogen/air flames, in: Twenty-Second Symposium (International) on Combustion, The Combustion Institute, 1988, pp. 1281-1290. 
[27] M. E. Kounalakis, J. P. Gore, G. M. Faeth, Mean and fluctuating radiation properties of nonpremixed turbulent carbon monoxide/air flames, ASME J Heat Transfer 111 (1989) 1021-1030.

[28] Y. R. Sivathanu, M. E. Kounalakis, G. M. Faeth, Soot and continuous radiation statistics of luminous turbulent diffusion flames, in: Twenty-Third Symposium (International) on Combustion, The Combustion Institute, 1990, pp. 1543-1550.

[29] M. Klassen, Y. R. Sivathanu, J. P. Gore, Simultaneous emission absorption-measurements in toluenefueled pool flames - mean and rms properties, Combust Flame 90 (1992) 34-44.

[30] Y. R. Sivathanu, J. P. Gore, Transient structure and radiation properties of strongly radiating buoyant flames, ASME J Heat Transfer 114 (1992) 659-665.

[31] Y. Zheng, R. S. Barlow, J. P. Gore, Spectral radiation properties of partially premixed turbulent flames, ASME J Heat Transfer 125 (2003) 1065-1073.

[32] Y. Zheng, R. S. Barlow, J. P. Gore, Measurements and calculations of spectral radiation intensities for turbulent non-premixed and partially premixed flames, ASME J Heat Transfer 125 (2003) 678-686.

[33] Y. Zheng, J. P. Gore, Measurements and inverse calculations of spectral radiation intensities of a turbulent ethylene/air jet flame, in: Thirtieth Symposium (International) on Combustion, The Combustion Institute, 2005, pp. 727-734.

[34] D. L. Blunck, M. E. H. C. L. Merkle, J. P. Gore, Influence of turbulent fluctuations on the radiation intensity emitted from exhaust plumes, J Thermoph Heat Transfer 26 (4) (2012) 581-589.

[35] D. Blunck, M. Harvazinski, B. Rankin, C. Merkle, J. Gore, Turbulent radiation statistics of exhaust plumes exiting from a subsonic axisymmetric nozzle, J Thermoph Heat Transfer 26 (2) (2012) 286-293.

[36] S. Mazumder, M. F. Modest, PDF modeling of turbulence radiation interactions, in: 1997 National Heat Transfer Conference, Baltimore, MD, ASME, 1997.

[37] S. Mazumder, M. F. Modest, Turbulence-radiation interactions in nonreactive flow of combustion gases, ASME J Heat Transfer 121 (1999) 726-729.

[38] G. Li, M. F. Modest, A PDF method to capture sharp gradients in turbulent heat transfer, in: Proceedings of the 2000 National Heat Transfer Conference, ASME, Pittsburgh, PA, 2000.

[39] G. Li, M. F. Modest, Application of composition PDF methods in the investigation of turbulence-radiation interactions, J Quant Spectrosc Radiat Transf 73 (2002) 461-472.

[40] A. Gupta, D. C. Haworth, M. F. Modest, Turbulence-radiation interactions in large-eddy simulations of luminous and nonluminous nonpremixed flames, Proc Comb Inst 34 (2013) 1281-1288.

[41] P. J. Coelho, Numerical simulation of the interaction between turbulence and radiation in reactive flows, Progr Energy Combust Sci 33 (4) (2007) 311-383.

[42] V. P. Kabashinikov, G. I. Myasnikova, Thermal radiation in turbulent flows-temperature and concentration fluctuations, Heat Transfer-Soviet Research 17 (6) (1985) 116-125.

[43] J. Y. Ko, H. K. Kim, T.-H. Song, Inversion of combustion gas temperature/concentration profile with radiation/turbulence interaction using SRS, J Quant Spectrosc Radiat Transf 110 (13) (2009) 1199-1206.

[44] P. Foster, Relation of time-mean transmission of turbulent flames to optical depth, Journal of the Institute of Fuel 42 (340) (1969) 179.

[45] M. F. Modest, Radiative Heat Transfer, 3rd Edition, Academic Press, New York, 2013. 
[46] A. D. Klose, U. Netz, J. Beuthan, A. H. Hielscher, Optical tomography using the time-independent equation of radiative transfer — part 1: forward model, J Quant Spectrosc Radiat Transf 72 (2002) 691-713.

[47] A. D. Klose, A. H. Hielscher, Optical tomography using the time-independent equation of radiative transfer — part 2: Inverse model, J Quant Spectrosc Radiat Transf 72 (2002) 715-732.

[48] A. Charette, J. Boulanger, H. K. Kim, An overview on recent radiation transport algorithm development for optical tomography imaging, J Quant Spectrosc Radiat Transf 109(17-18) (2008) 2743-2766.

[49] J. Nocedal, S. J. Wright, Numerical optimization, 2nd Edition, Springer Verlag, Berlin, 2006.

[50] W. H. Press, S. A. Teukolsky, W. T. Vetterling, B. P. Flannery, Numerical Recipies in FORTRAN - The Art of Scientific Computing, 2nd Edition, Cambridge University Press, Cambridge, 1992.

[51] T. Ren, M. F. Modest, Optical determination of temperature and species concentration for homogeneous turbulent gas medium, Intl J Heat Mass Transfer 90 (2015) 1178-1187.

[52] L. S. Rothman, I. E. Gordon, R. J. Barber, H. Dothe, R. R. Gamache, A. Goldman, V. I. Perevalov, S. A. Tashkun, J. Tennyson, HITEMP, the high-temperature molecular spectroscopic database, J Quant Spectrosc Radiat Transf 111 (15) (2010) 2139-2150.

[53] M. F. Modest, S. P. Bharadwaj, High-resolution, high-temperature transmissivity measurements and correlations for carbon dioxide-nitrogen mixtures, J Quant Spectrosc Radiat Transf 73 (2-5) (2002) 329-338.

[54] S. P. Bharadwaj, M. F. Modest, Medium resolution transmission measurements of $\mathrm{CO}_{2}$ at high temperature - an update, J Quant Spectrosc Radiat Transf 103 (2007) 146-155.

[55] V. Evseev, A. Fateev, S. Clausen, High-resolution transmission measurements of $\mathrm{CO}_{2}$ at high temperatures for industrial applications, J Quant Spectrosc Radiat Transf 113 (2012) 2222-2233.

[56] S. P. Bharadwaj, M. F. Modest, R. J. Riazzi, Medium resolution transmission measurements of water vapor at high temperature, ASME J Heat Transfer 128 (2006) 374-381.

[57] A. Fateev, S. Clausen, On-line non-contact gas analysis, Danmarks Tekniske Universitet, Ris $\varnothing$ Nationallaboratoriet for Bæredygtig Energi, 2008.

[58] F. Kritzstein, A. Soufiani, Infrared gas radiation from a homogeneously turbulent medium, Intl J Heat Mass Transfer 36 (7) (1993) 1749-1762.

[59] L. Soucasse, P. Rivière, A. Soufiani, Subgrid-scale model for radiative transfer in turbulent participating media, J Comp Phys 257 (2014) 442-459.

[60] B. Khorsandi, L. Mydlarski, S. Gaskin, Noise in turbulence measurements using acoustic doppler velocimetry, Journal of Hydraulic Engineering 138 (10) (2012) 829-838.

[61] C. Sardu, D. Lasagna, G. Iuso, Noise filtering for wall-pressure fluctuations in measurements around a cylinder with laminar and turbulent flow separation, Journal of Fluids Engineering 138 (6) (2016) 061101.

[62] D. H. Lenschow, L. Kristensen, Uncorrelated noise in turbulence measurements, Journal of Atmospheric and Oceanic Technology 2 (1) (1985) 68-81.

[63] R. S. Barlow, International workshop on measurement and computation of turbulent nonpremixed flames (TNF), website: http://www.sandia.gov/TNF/abstract.html.

[64] C. B. da Silva, I. Malico, P. J. Coelho, Radiation statistics in homogeneous isotropic turbulence, New Journal of Physics 11 (9) (2009) 093001.

[65] M. N. Ozisik, Inverse heat transfer: fundamentals and applications, CRC Press, 2000. 